\title{
High-Order Local Rate of Convergence By Mesh-Refinement in the Finite Element Method
}

\author{
By Kenneth Eriksson
}

\begin{abstract}
We seek approximations of the solution $u$ of the Neumann problem for the equation $L u=f$ in $\Omega$ with special emphasis on high-order accuracy at a given point $x_{0} \in \bar{\Omega}$. Here $\Omega$ is a bounded domain in $R^{N}(N \geqslant 2)$ with smooth boundary, and $L$ is a second-order, uniformly elliptic, differential operator with smooth coefficients. An approximate solution $u_{h}$ is determined by the standard Galerkin method in a space of continuous piecewise polynomials of degree at most $r-1$ on a partition $\Delta_{h}\left(x_{0}, \alpha\right)$ of $\Omega$. Here $h$ is a global mesh-size parameter, and $\alpha$ is the degree of a certain systematic refinement of the mesh around the given point $x_{0}$, where larger $\alpha$ 's mean finer mesh, and $\alpha=0$ corresponds to the quasi-uniform case with no refinement. It is proved that, for suitable (sufficiently large) $\alpha$ 's the high-order error estimate $\left(u-u_{h}\right)\left(x_{0}\right)=O\left(h^{2 r-2}\right)$ holds. A corresponding estimate with the same order of convergence is obtained for the first-order derivatives of $u-u_{h}$. These estimates are sharp in the sense that the required degree of refinement in each case is essentially the same as is needed for the local approximation to this order near $x_{0}$. For the estimates to hold, it is sufficient that the exact solution $u$ have derivatives to the $r$ th order which are bounded close to $x_{0}$ and square integrable in the rest of $\Omega$. The proof of this uses high-order negative-norm estimates of $u-u_{h}$. The number of elements in the considered partitions is of the same order as in the corresponding quasi-uniform ones. Applications of the results to other types of boundary value problems are indicated.
\end{abstract}

0. Introduction. Let $\Omega$ be a bounded domain in $R^{N}, N \geqslant 2$, with smooth boundary $\partial \Omega$ and consider the Neumann problem to find $u$ such that

$$
\begin{gathered}
L u=-\sum_{i, j=1}^{N} \frac{\partial}{\partial x_{j}}\left(a_{i j} \frac{\partial u}{\partial x_{i}}\right)+\sum_{i=1}^{N} a_{i} \frac{\partial u}{\partial x_{i}}+a u=f \text { in } \Omega, \\
\frac{\partial u}{\partial n_{c}}=\sum_{i, j=1}^{N} a_{i j} \frac{\partial u}{\partial x_{i}} n_{j}=0 \quad \text { on } \partial \Omega .
\end{gathered}
$$

Here $L$ is assumed to be uniformly elliptic with smooth coefficients, $n=\left(n_{j}\right)$ and $n_{c}$ denote the exterior normal and conormal to $\partial \Omega$, respectively. Assume also that the bilinear form

$$
A(v, w)=\int_{\Omega}\left\{\sum_{i, j=1}^{N} a_{i j} \frac{\partial v}{\partial x_{i}} \frac{\partial w}{\partial x_{j}}+\sum_{i=1}^{N} a_{i} \frac{\partial v}{\partial x_{i}} w+a v w\right\} d x
$$

Received September 22, 1983; revised July 23, 1984.

1980 Mathematics Subject Classification. Primary 65N30.

Key words and phrases. Finite element method, Galerkin approximation, mesh-refinement, high-order local convergence, Neumann problem. 
associated with $L$ is coercive over $H^{1}(\Omega)$; that is, for some constant $c>0$,

$$
A(v, v) \geqslant c\|v\|_{H^{1}(\Omega)}^{2} \quad \forall v \in H^{1}(\Omega) .
$$

In order to approximate $u$, let $\left\{S_{h}\right\}, h$ small and positive, be a one-parameter family of finite-dimensional subspaces of $H^{1}(\Omega)$ and define the Galerkin approximation $u_{h} \in S_{h}$ of $u$ by

$$
A\left(u_{h}, v_{h}\right)=\left(f, v_{h}\right) \quad \forall v_{h} \in S_{h},
$$

where $(\cdot, \cdot)$ is the standard inner product in $L_{2}(\Omega)$. Multiplication of $(0.1)$ by $v_{h}$ and integration by parts, using $(0.2)$, shows that $(0.3)$ holds for the exact solution $u$. Hence,

$$
A\left(u-u_{h}, v_{h}\right)=0 \quad \forall v_{h} \in S_{h} .
$$

Therefore, we shall also refer to $u_{h}$ as the " $A$-projection" of $u$.

In this paper, $S_{h}$ will consist of the restrictions to $\bar{\Omega}$ of all continuous piecewise polynomials of degree at most $r-1$ on a simplicial mesh, with $h$ the maximal diameter of the simplices. From the approximation properties of $S_{h}$, the a priori estimate

$$
\left\|u-u_{h}\right\|_{H^{1}(\Omega)} \leqslant C h^{r-1}\|u\|_{H^{r}(\Omega)}
$$

is easily obtained. Using the Aubin-Nitsche lemma (see [7, p. 137]), one can derive a sharper estimate in the $L_{2}(\Omega)$-norm: namely,

$$
\left\|u-u_{h}\right\|_{L_{2}(\Omega)} \leqslant C h^{r}\|u\|_{H^{r}(\Omega)} .
$$

Optimal, or nearly optimal, a priori error estimates can be attained also in the maximum norm, but require a more sophisticated analysis. With an additional nondegeneracy condition on the elements, one can prove that

$$
\left\|u-u_{h}\right\|_{L_{\infty}(\Omega)} \leqslant C(\ln (1 / h))^{\bar{r}} h^{r}\|u\|_{W_{\infty}^{r}(\Omega)},
$$

where $\bar{r}=1$ if $r=2, \bar{r}=0$ for $r>2$. In the case of $N=2$ and for a special form $A$, a proof of this was given by Scott in [19]. For a survey on related results where other boundary value problems are also considered, see Nitsche [11].

In this paper we shall consider a simplicial mesh which has been refined in a certain way close to a given fixed point $x_{0} \in \bar{\Omega}$. We shall then be able to show higher-order convergence at $x_{0}$. Our main result will be

$$
\left|\left(u-u_{h}\right)\left(x_{0}\right)\right| \leqslant C h^{2 r-2}\|u\|_{W_{\infty}^{r}(\Omega)} .
$$

This holds provided the degree of the prescribed refinement is such that the elements closest to $x_{0}$ have diameters of order $h^{2-2 / r+\varepsilon}$ for some $\varepsilon>0$. In the case $\varepsilon=0$, (0.6) holds with a logarithmic modification. It is clear that only the case $r>2$ is of interest in this context, even though, when $r=2$ and $\varepsilon>0,(0.6)$ is slightly better than $(0.5)$ as an estimate for $\left(u-u_{h}\right)\left(x_{0}\right)$. We shall also demonstrate that if the refinement is such that the elements closest to $x_{0}$ have diameters of order $h^{2+\varepsilon}$, then the same bound as in (0.6) can be obtained for the gradient of $u-u_{h}$ :

$$
\left|\nabla\left(u-u_{h}\right)\left(x_{0}\right)\right| \leqslant C h^{2 r-2}\|u\|_{W_{\infty}^{r}(\Omega)} .
$$


This result is of interest also for $r=2$. Next, by localizing the arguments to a neighborhood $\Omega^{0} \subset \Omega$ of $x_{0}$, we derive the estimate

$$
\left|\left(u-u_{h}\right)\left(x_{0}\right)\right| \leqslant C h^{2 r-2}\|u\|_{W_{\infty}^{r}\left(\Omega^{0}\right)}+C\left\|u-u_{h}\right\|_{-m, \Omega^{0}}^{*},
$$

where $\|\cdot\|_{-m, \Omega^{0}}^{*}$ denotes a weak negative norm to be defined in Section 9. Using known high-order error estimates for $u-u_{h}$ in such negative norms, this will show

$$
\left|\left(u-u_{h}\right)\left(x_{0}\right)\right| \leqslant C h^{2 r-2}\left\{\|u\|_{W_{\infty}^{r}\left(\Omega^{0}\right)}+\|u\|_{H^{r}(\Omega)}\right\},
$$

so that the regularity requirement on $u$ away from $x_{0}$ can be relaxed, with a high rate of convergence retained at $x_{0}$. The corresponding results are shown for the gradient of $u-u_{h}$. Since $(0.8)$ and its counterpart for the gradient require only local information on $u-u_{h}$, our results can also be applied in other situations where negative-norm estimates are known: for example, in connection with various procedures for treating the Dirichlet problem. The above results suggest that the Galerkin solution is a fairly local approximator of $u$ and that pollution effects are moderate, at least for regular problems.

The assumptions on the refinement are that the mesh size decays toward $x_{0}$ approximately as $h$ times some power $\alpha$ of the distance, and that local inverse estimates hold. As is seen from the above discussion, the degree $\alpha$ of refinement needed for our results is essentially the same as is required for local approximation to this high order near $x_{0}$. Hence, our results are sharp in this sense. Furthermore, it has been shown in Eriksson [5] that the refinement does not seriously increase the total number of elements in the partition, so that the amount of work needed to solve for $u_{h}$ is essentially the same as for a quasi-uniform mesh.

One may argue that a weak point about the mesh-refinement procedure is that different grids are needed for points $x_{0}$ at different locations. In practice, however, it is often the case that one is primarily interested in the solution only in the neighborhood of one specific point. For instance, in fracture mechanics one often knows a priori the point which is the most critical one for the structure and wants detailed information about the solution near this point. On the other hand, if more than one point is of interest, one may, of course, use a mesh which has been locally refined around each one of the points under consideration. Our analysis does not quite cover this case, but it is reasonable to believe that, under the appropriate assumptions, one then obtains $O\left(h^{2 r-2}\right)$ convergence at all the centers of refinement simultaneously, using the same one mesh. Let us also mention that there exist commercial codes for automatic generation of meshes with local refinements like the ones we consider here.

Mesh-refinements have previously been used in connection with elliptic problems in order to enable functions in $S_{h}$ to fit known singularities in the exact solution properly. See, for example, Schatz and Wahlbin [15], where the singularities of $u$ are caused by the corners of the domain. For a singular two-point boundary value problem, see Schreiber [18]. In Eriksson [5], [6] mesh-refinements were used in order to approximate Green's function to a high-order of accuracy. The idea in these papers is very much related to the present work. 
As mentioned above, $O\left(h^{2 r-2}\right)$ estimates are known in many cases for $u-u_{h}$ as measured in a suitable negative norm; see Bramble and Osborn [3]. Pointwise superconvergence at mesh points is common for one-dimensional problems. In higher dimensions, $O\left(h^{2 r-2}\right)$ error estimates have been obtained by various averaging procedures applied to an already existing approximation $u_{h}$ of $u$; see Bramble and Schatz [4], Louis and Natterer [10], Thomée [20], and references therein. Here the post-processing procedure proposed by Bramble and Schatz is easy to perform, but requires a uniform mesh and can, therefore, in general, only give interior results. On the other hand, the method suggested by Louis and Natterer requires that a fundamental solution be a priori known, which, in practice, limits the possibility of application to the case of constant coefficients and no lower-order terms.

The methods of proof employed here are reminiscent of those used by Schatz and Wahlbin in a series of papers: [13], [14], [15], [16]. Let $\mathscr{G}$ be the Green function for (0.1) and (0.2), and let $\mathscr{G}_{h} \in S_{h}$ be its $A^{*}$-projection. Then the starting point for the proof of our estimate $(0.6)$ will be

$$
\left(u-u_{h}\right)\left(x_{0}\right)=A\left(u-\chi_{h}, \mathscr{G}-\mathscr{G}_{h}\right) .
$$

Here $\chi_{h}$ can be any function in $S_{h}$, and therefore, by the approximation properties of $S_{h}$ due to the refinement, the essential step in the proof will be to estimate $\mathscr{G}_{-} \mathscr{G}_{h}$ in a weighted $W_{1}^{1}$-norm. An important tool for this will be the use of local $H^{1}$-estimates similar to those obtained by Nitsche and Schatz in [12]. In fact, to avoid difficulties at the point $x_{0}$, we shall not use the exact Green function but a smooth approximation of it.

An outline of the paper is as follows. Notation and some results on elliptic regularity are given in Sections 1 and 2. The exact hypotheses on the mesh-refinements are presented in Section 3, and some desired properties of the corresponding piecewise-polynomial spaces are presented in Section 4.

In Section 5 , the main result $(0.6)$ is stated in precise terms and then proved. Section 6 is devoted to the proof of the weighted $W_{1}^{1}$-norm estimate mentioned above. The improved convergence ( 0.7$)$ for the gradient is proved in Section 7 . For completeness, a negative-norm estimate referred to in the later sections is derived in Section 8 . The local estimate $(0.8)$ is obtained in Section 9 and its counterpart for the gradient, in Section 10.

1. Notation and Preliminaries. For a domain $\Omega^{\prime} \subset R^{N}$ and $m$ a nonnegatve integer, $W_{q}^{m}\left(\Omega^{\prime}\right)$ will denote the usual Sobolev space with norm

$$
\|v\|_{W_{q}^{m^{\prime}\left(\Omega^{\prime}\right)}}= \begin{cases}\left\{\sum_{|\beta| \leqslant m}\left\|D^{\beta} v\right\|_{L_{q}\left(\Omega^{\prime}\right)}^{q}\right\}^{1 / q} & \text { if } 1 \leqslant q<\infty, \\ \max _{|\beta| \leqslant m}\left\|D^{\beta} v\right\|_{L_{\iota^{\prime}}\left(\Omega^{\prime}\right)} & \text { if } q=\infty,\end{cases}
$$

where $\beta=\left(\beta_{1}, \ldots, \beta_{N}\right)$ with $\beta_{i}$ nonnegative integers, $|\beta|=\sum_{i=1}^{N} \beta_{i}$. $D^{\beta}=D_{x}^{\beta}=$ $\left(\partial / \partial x_{1}\right)^{\beta_{1}} \cdots\left(\partial / \partial x_{N}\right)^{\beta_{N}}$, and $\|\cdot\|_{L_{q}\left(\Omega^{\prime}\right)}$ is the norm in the Banach space $L_{q}\left(\Omega^{\prime}\right)$. We shall also use the seminorm $|\cdot|_{W_{q}^{m}\left(\Omega^{\prime}\right)}$, defined as the corresponding norm, but with $\beta$ ranging over $|\beta|=m$ only. For $q=2$ we write $W_{2}^{m}\left(\Omega^{\prime}\right)=H^{m}\left(\Omega^{\prime}\right)$, and for the corresponding norm and seminorm, $\|\cdot\|_{W_{2}^{m}\left(\Omega^{\prime}\right)}=\|\cdot\|_{H^{m}\left(\Omega^{\prime}\right)}=\|\cdot\|_{m, \Omega^{\prime}}$ and $|\cdot|_{W_{2}^{m}\left(\Omega^{\prime}\right)}=|\cdot|_{m, \Omega^{\prime}}$, respectively. 
The space of restrictions to $\bar{\Omega}^{\prime}$ of infinitely differentiable functions on $R^{N}$ will be denoted $C^{\infty}\left(\Omega^{\prime}\right)$, and $C_{0}^{\infty}\left(\Omega^{\prime}\right)$ will consist of all functions in $C^{\infty}\left(\Omega^{\prime}\right)$ with compact support in $\Omega^{\prime}$.

For $m \geqslant 0, m$ an integer, we define the negative norms $\|\cdot\|_{-m, \Omega^{\prime}}$ and $\|\cdot\| \|_{-m, \Omega^{\prime}}$ by

$$
\|v\|_{-m, \Omega^{\prime}}=\sup _{w \in C_{0}^{\infty}\left(\Omega^{\prime}\right)} \frac{(v, w)_{\Omega^{\prime}}}{\|w\|_{m, \Omega^{\prime}}}
$$

and

$$
\|v\|_{-m, \Omega^{\prime}}=\sup _{w \in C^{\infty}\left(\Omega^{\prime}\right)} \frac{(v, w)_{\Omega^{\prime}}}{\|w\|_{m, \Omega^{\prime}}}
$$

where $(v, w)_{\Omega^{\prime}}=\int_{\Omega^{\prime}} v w d x$.

Throughout this paper we shall let $\Omega$ be a fixed bounded domain in $R^{N}, N \geqslant 2$, with $C^{\infty}$ boundary $\partial \Omega$. When $\Omega^{\prime}=\Omega$ we shall shorten the notation and write $H^{m}(\Omega)=H^{m},\|\cdot\|_{m, \Omega}=\|\cdot\|_{m},(\cdot, \cdot)_{\Omega}=(\cdot, \cdot)$, etc.

For $\Omega^{\prime} \subset \Omega$ we shall consider the bilinear form $A_{\Omega^{\prime}}=A(\cdot, \cdot)_{\Omega^{\prime}}$, defined by

$$
A(v, w)_{\Omega^{\prime}}=\int_{\Omega^{\prime}}\left\{\sum_{i, j=1}^{N} a_{i j} \frac{\partial v}{\partial x_{i}} \frac{\partial w}{\partial x_{j}}+\sum_{i=1}^{N} a_{i} \frac{\partial v}{\partial x_{i}} w+a v w\right\} d x,
$$

where we require that the coefficients $a_{i j}, a_{i}$, and $a$ be functions in $C^{\infty}(\Omega)$ satisfying $a_{i j}=a_{j i}$. Although we shall mainly be interested in the form $A=A_{\Omega}$, we make the following assumption: There is a constant $c_{A}>0$ such that

$$
A(v, v)_{\Omega^{\prime}} \geqslant c_{A}\|v\|_{1, \Omega^{\prime}}^{2} \quad \forall v \in H^{1}\left(\Omega^{\prime}\right), \forall \Omega^{\prime} \subset \Omega .
$$

The reason for our interest in the bilinear form $A$ is its connection with the Neumann problem for the second-order partial differential equation

$$
-\sum_{i, j=1}^{N} \frac{\partial}{\partial x_{j}}\left(a_{i j} \frac{\partial u}{\partial x_{i}}\right)+\sum_{i=1}^{N} a_{i} \frac{\partial u}{\partial x_{i}}+a u=f
$$

Moreover, our main result will have applications to other boundary value problems for this equation, such as the Dirichlet problem.

We shall later need the fact that the coefficients $a_{i j}$ satisfy the following condition: There is a constant $c_{A}>0$ such that

$$
\sum_{i, j=1}^{N} a_{i j}(x) \lambda_{i} \lambda_{j} \geqslant c_{A}|\lambda|^{2} \quad \forall \lambda \in R^{N}, \forall x \in \bar{\Omega} ;
$$

i.e., the associated differential equation is uniformly elliptic. This is, in fact, not an extra assumption on the coefficients $a_{i j}$, but follows easily from (1.2) with the same constant $c_{A}$. Indeed, take $\Omega^{\prime}=\{x \in \Omega:|x-\bar{x}|<\varepsilon\}$ and $v=m_{\varepsilon}^{-1 / 2} \lambda \cdot(x-\bar{x})$, where $m_{\varepsilon}=\int_{\Omega^{\prime}} d x$. Then, since $a_{i j}$ are smooth by assumption, a simple computation shows that then for small $\varepsilon$,

$$
A(v, v)_{\Omega^{\prime}}=\sum_{i, j=1}^{N} a_{i}(\bar{x}) \lambda_{i} \lambda_{j}+O(\varepsilon)
$$

and

$$
\|v\|_{1, \Omega^{\prime}}^{2}=|\lambda|^{2}+O\left(\varepsilon^{2}\right)
$$

Inserting this into (1.2) and letting $\varepsilon$ tend to zero yields the desired inequality (1.3). 
Remark 1.1. Assumption (1.2) is unnecessarily restrictive for the purposes of this paper. In fact, the following global coercivity condition suffices: There is a constant $c_{A}^{\prime}>0$ such that

$$
A(v, v)_{\Omega} \geqslant c_{A}^{\prime}\|v\|_{1, \Omega}^{2} \quad \forall v \in H^{1}(\Omega) .
$$

In Sections 9 and 10 , where (1.2) is used for $\Omega^{\prime} \neq \Omega$, it is possible to employ instead the modified bilinear form $A_{k, \Omega^{\prime}}(v, w)=A(v, w)_{\Omega^{\prime}}+k(v, w)_{\Omega^{\prime}}$, which satisfies (1.2) if $k$ is sufficiently large (cf. Appendix 1 of [13]).

We end this section with a list of some further notation and definitions. We shall write

$$
\begin{gathered}
m(K)=\int_{K} d x, \quad \operatorname{diam}(K)=\sup _{x, y \in K}|x-y|, \\
\operatorname{dist}\left(K, K^{\prime}\right)=\inf _{x \in K, y \in K^{\prime}}|x-y|, \quad \text { and } B_{d}(y)=\left\{x \in R^{N}:|x-y|<d\right\} .
\end{gathered}
$$

Moreover, $P_{r}(K)$ will be the set of all polynomials of degree $\leqslant r$ restricted to $K$. Throughout the paper we shall use the letters $c$ and $C$ to denote various positive constants. For $f$ and $g$ two positive functions, we shall write $f \sim g$ if there are constants $c$ and $C$ such that $f \geqslant c g$ and $f \leqslant C g$. We also define the unit $N$-simplex $T$ by

$$
T=\left\{x \in R^{N}: x_{i} \geqslant 0, i=1, \ldots, N, \text { and } \sum_{i=1}^{N} x_{i} \leqslant 1\right\} .
$$

An arbitrary $N$-simplex is obtained by a nonsingular affine transformation of $T$. A face of a simplex is any one of the $N+1(N-1)$-simplices constituting its boundary.

2. Elliptic Regularity. In this section we shall be concerned with the variational problem

$$
A(G, v)_{\Omega^{\prime}}=(g, v)_{\Omega^{\prime}}, \quad \forall v \in H^{1}\left(\Omega^{\prime}\right),
$$

and with the regularity of the solution $G$ in terms of $g$.

By the boundedness of the coefficients, the form $A_{\Omega^{\prime}}$ is continuous on $W_{p}^{1}\left(\Omega^{\prime}\right) \times$ $W_{q}^{1}\left(\Omega^{\prime}\right)$ for $1 \leqslant p, q \leqslant \infty$ and $p^{-1}+q^{-1}=1$. In fact, by Hölder's inequality, there is a constant $C_{A}$, independent of $\Omega^{\prime}$, such that

$$
A(v, w)_{\Omega^{\prime}} \leqslant C_{A}\|v\|_{W_{p}^{1}\left(\Omega^{\prime}\right)}\|w\|_{W_{q}^{1}\left(\Omega^{\prime}\right)} \quad \forall(v, w) \in W_{p}^{1}\left(\Omega^{\prime}\right) \times W_{q}^{1}\left(\Omega^{\prime}\right) .
$$

In particular, the form is continuous on $H^{1}\left(\Omega^{\prime}\right)$. We shall need (2.2) for $p=2$ and $p=\infty$ only.

The results of the following lemma are special cases of the Lax-Milgram lemma (cf., e.g., Ciarlet [7]).

LEMMA 2.1. Let the coefficients of the bilinear form $A$ be such that (1.2) and (2.2) ( with $p=2$ ) hold. Then, for given $\Omega^{\prime} \subset \Omega$ and $g \in L_{2}\left(\Omega^{\prime}\right)$, there is a unique $G \in H^{1}\left(\Omega^{\prime}\right)$ satisfying (2.1). Furthermore, given any $G \in H^{1}\left(\Omega^{\prime}\right)$ and a closed subspace $S$ of $H^{1}\left(\Omega^{\prime}\right)$, the $A_{\Omega^{\prime}}$-projection of $G$ on $S$ is well-defined; i.e., there exists a unique $G_{S} \in S$ for which

$$
A\left(G-G_{S}, v_{S}\right)_{\Omega^{\prime}}=0 \quad \forall v_{S} \in S
$$


For $\Omega^{\prime}$ with a sufficiently smooth boundary $\partial \Omega^{\prime}$ we have the following well-known, elliptic, regularity result (cf., e.g., Schechter [17]).

LEMMA 2.2. Let the coefficients of the form $A$ be as in Section 1 and assume that (1.2) holds. Furthermore, let $\Omega^{\prime} \subset \Omega$ have a $C^{\infty}$ boundary $\partial \Omega^{\prime}$, and let $s \geqslant 0$ be an integer. Then there is a constant $C$ such that, if $g \in H^{s}\left(\Omega^{\prime}\right)$ and $G$ is defined by (2.1), then

$$
\|G\|_{s+2, \Omega^{\prime}} \leqslant C\|g\|_{s, \Omega^{\prime}}
$$

We shall make extensive use of local estimates on $G$ away from the support of $g$. We have the following lemma:

LeMma 2.3. Let the assumptions be as in Lemma 2.2, let $s>0$, and let $\beta$ be any multi-index of length $|\beta| \leqslant s$. Then there is a constant $C$ such that the following holds: Let $g \in L_{2}\left(\Omega^{\prime}\right)$, let $x \in \Omega^{\prime}$, and set $R=\operatorname{dist}(x, \operatorname{supp}(g))$. Then, for $G$ defined by (2.1),

$$
\left|D^{\beta} G(x)\right| \leqslant C R^{-N+2-s}\|g\|_{L_{1}\left(\Omega^{\prime}\right)} .
$$

Proof. The solution $G$ of $(2.1)$ is given by

$$
G(x)=\int_{\Omega^{\prime}} \mathscr{G}(x, y) g(y) d y,
$$

where $\mathscr{G}(x, y)$ is the Green function. It is known (cf., e.g., Krasovskii [9]) that

$$
\left|D_{x}^{\beta} \mathscr{G}(x, y)\right| \leqslant C|x-y|^{-N+2-s} \text {. }
$$

Differentiation of (2.5) under the integral sign, application of Hölder's inequality, and (2.6) give

$$
\begin{aligned}
\left|D^{\beta} G(x)\right| & =\left|\int_{\Omega^{\prime}} D_{x}^{\beta} \mathscr{G}(x, y) g(y) d y\right| \\
& \leqslant\left\|D_{x}^{\beta} \mathscr{G}(x, \cdot)\right\|_{L_{\infty}(\operatorname{supp}(g))}\|g\|_{L_{1}\left(\Omega^{\prime}\right)} \leqslant C R^{-N+2-s}\|g\|_{L_{1}\left(\Omega^{\prime}\right)},
\end{aligned}
$$

which was to be proved.

We finally note that in all of this section we could have considered the adjoint problem as well. That is, for $g \in L_{2}\left(\Omega^{\prime}\right)$, there is a $G \in H^{1}\left(\Omega^{\prime}\right)$ (in general, different from our previous $G$ ) such that

$$
A(v, G)_{\Omega^{\prime}}=(v, g)_{\Omega^{\prime}} \quad \forall v \in H^{1}\left(\Omega^{\prime}\right),
$$

and the regularity results above hold for this $G$ as well. Moreover, the $A_{\Omega^{\prime}}^{*}$-projection $G_{S} \in S$ of $G$ is well-defined by

$$
A\left(v_{S}, G-G_{S}\right)_{\Omega^{\prime}}=0 \quad \forall v_{S} \in S .
$$

3. Mesh-Refinement Around a Point. In this section we define certain partitions of $\Omega$ which are refined around a given fixed point $x_{0} \in \bar{\Omega}$. To these partitions there will correspond finite-dimensional function spaces, to be introduced in Section 4.

We shall divide $\Omega$ into simplices, which will be modified at the boundary $\Omega$, since the simplices cannot fit exactly the curved parts of $\partial \Omega$. For a small positive parameter $h$, we shall consider a family of such partitions defined as follows: Let $\Omega$ be covered by the union $\hat{\Omega}_{h}$ of the simplices $\hat{\tau}_{i}^{h}, i=1, \ldots, I(h)$, where we require that 
the $\hat{\tau}_{i}^{h}$ have disjoint interiors and satisfy the standard assumption that, for $i=$ $1, \ldots, I(h)$, any face of $\hat{\tau}_{i}^{h}$ is either a part of $\partial \hat{\Omega}_{h}$ or corresponds exactly to a face of an adjacent simplex. As our partition we take the sets, with $\operatorname{int}(\cdot)=$ the interior of $(\cdot)$,

$$
\tau_{i}^{h}=\operatorname{int}\left(\hat{\tau}_{i}^{h} \cap \Omega\right), \quad i=1, \ldots, I(h) .
$$

The desired features of the partition are determined by the following two require-

- ments: For some fixed positive constants $c_{S}, c_{R}$, and $C_{R}$, for $x_{0} \in \bar{\Omega}$, for $\alpha$ with $0 \leqslant \alpha<1$, and for $h$ small and positive, we have

$$
\begin{gathered}
m\left(\tau_{i}^{h}\right) \geqslant c_{S} \operatorname{diam}^{N}\left(\hat{\tau}_{i}^{h}\right), \quad i=1, \ldots, I(h), \\
c_{R} \operatorname{diam}\left(\tau_{i}^{h}\right) \leqslant h \sup _{x \in \tau_{i}^{h}}\left|x-x_{0}\right|^{\alpha} \leqslant C_{R} \operatorname{diam}\left(\tau_{i}^{h}\right), \quad i=1, \ldots, I(h) .
\end{gathered}
$$

We use the notation $\Delta_{h}\left(x_{0}, \alpha\right)$ for partitions obtained as above and satisfying (SA) and (RA).

The "shape" assumption (SA) implies, on the one hand, that the simplices do not degenerate, and, on the other hand, near the boundary, that a fixed minimum portion of each simplex is inside $\Omega$. This can be restated in the following equivalent but more useful way: There are fixed positive constants $c_{S}^{\prime}$ and $C_{S}^{\prime}$ and, for each $\tau=\tau_{i}^{h}$, a nonsingular affine mapping $A_{\tau}$ with $A_{\tau}(x)=B_{\tau} x+b_{\tau}, B_{\tau}=\left(b_{i j}\right), B_{\tau}^{-1}=$ $\left(b_{i j}^{\prime}\right)$, such that

$(\mathrm{SA})^{\prime} \quad c_{S}^{\prime} T \subset A_{\tau}(\tau) \subset A_{\tau} \hat{\tau}=T, \quad\left|b_{i j}\right| \leqslant C_{S}^{\prime} \operatorname{diam}^{-1}(\tau), \quad\left|b_{i j}^{\prime}\right| \leqslant C_{S}^{\prime} \operatorname{diam}(\tau)$.

The refinement assumption $(\mathrm{RA})=(\mathrm{RA})_{x_{0}, \alpha}$ roughly says that an element at distance $r$ from $x_{0}$ has diameter approximately equal to $h r^{\alpha}$. Since $\Omega$ is bounded, the global mesh size is bounded by $C h$. Elements close to $x_{0}$ are forced to have diameter of order $h^{1 /(1-\alpha)}$. Hence, the parameter $\alpha$ is a measure of the refinement.

For a discussion of the possibility of constructing a mesh satisfying (SA) and (RA), we refer to Eriksson [5]. There, it is also shown that, for a fixed $\alpha<1$, the total number of elements in such a partition is proportional to $h^{-N}$ as in the quasiuniform case. Hence, the size of the finite element matrix and, thereby, the amount of work required to solve for the Galerkin approximation are not seriously increased.

For our proofs we shall need various subsets of $\Omega$. For integers $j$ we define

$$
\begin{array}{ll}
\Omega_{j}=\Omega \cap B_{j} & \text { where } B_{j}=B_{d_{j}}\left(x_{0}\right), d_{j}=2^{-j}, \\
D_{j}=\Omega \cap A_{j} & \text { where } A_{j}=B_{j} \backslash \bar{B}_{j+1},
\end{array}
$$

and

$$
D_{j}^{k}=D_{j-k} \cup \cdots \cup D_{j+k}, \quad k=0,1,2, \ldots
$$

In the technical work below we shall need to know that the local mesh size on $D_{j}$ is small in relation to $d_{j}$, in a sense to be made precise later. This will turn out to be the case for all $j \leqslant J_{1}$ if we define the function $J_{1}=J_{1}(h)=J_{1}(h, \alpha)$ by

$$
2^{-J_{1}}=C_{1} h^{1 /(1-\alpha)}
$$

where the constant $C_{1}$ is chosen sufficiently large. The significance of the quantity $J_{1}$ will be clear in Section 4 , where we shall also fix $C_{1}$. Defining $h_{j}=h d_{j}^{\alpha}$, it follows 
easily from (RA) that, for $j \leqslant J_{1}, h_{j}$ is proportional to the local mesh size on $D_{j}$, and that

$$
h_{j} d_{j}^{-1}=h d_{j}^{\alpha-1} \leqslant h\left(C_{1} h^{1 /(1-\alpha)}\right)^{\alpha-1}=1 / C_{1}^{1-\alpha},
$$

which also expresses, if $C_{1}$ is large, the relative smallness of the mesh on $D_{j}$. We finally note that, since $\Omega$ is bounded, the sets $D_{j}$ will be empty for $j$ less than some integer $j_{1}$.

4. Spaces. Inverse Estimates. Approximation Properties. Let $\Delta_{h}=\Delta_{h}\left(x_{0}, \alpha\right)$ be a partition as described in Section 3, and let $r$, with $r \geqslant 2$, be an integer. We shall denote by $S_{h}=S_{h}\left(r, x_{0}, \alpha\right)$ the finite-dimensional space of functions in $C(\bar{\Omega})$ which reduce to polynomials in $P_{r-1}$ in each of the elements $\tau=\tau^{h} \in \Delta_{h}$. Moreover, if $\Omega^{\prime} \subset \Omega$, the space of all restrictions to $\bar{\Omega}^{\prime}$ of functions in $S_{h}$ will be written $S_{h}\left(\Omega^{\prime}\right)$, and $S_{h}^{0}\left(\Omega^{\prime}\right)$ will consist of all functions in $S_{h}$ vanishing outside $\bar{\Omega}^{\prime}$. We shall use the notation $\hat{\chi}_{h}$ for the obvious piecewise-polynomial extension to $\hat{\Omega}_{h}$ of a function $\chi_{h} \in S_{h}$; we use $\hat{S}_{h}$ for the corresponding space.

The following inverse estimate is standard for interior elements.

Proposition 4.1. Let the partition $\Delta_{h}$ and the corresponding piecewise-polynomial space $S_{h}$ be as above. Then there is a constant $C_{2}$, depending only on $N, r, c_{S}^{\prime}$, and $C_{S}^{\prime}$, such that, for $1 \leqslant p, q \leqslant \infty$, integers $0 \leqslant t<s \leqslant r, v_{h} \in S_{h}$, and any element $\tau \in \Delta_{h}$, the following holds:

$$
\left|v_{h}\right|_{W_{p}^{s}(\tau)} \leqslant C_{2}[\operatorname{diam}(\tau)]^{t-s-N\left(q^{-1}-p^{-1}\right)}\left|v_{h}\right|_{w_{q}^{t}(\tau)}
$$

Furthermore, if $m>0$ is an integer, there is a constant $C_{3}=C_{3}\left(N, m, c_{S}^{\prime}, C_{S}^{\prime}\right)$ such that, for $v_{h} \in S_{h}$ and any element $\tau$,

$$
\left\|v_{h}\right\|_{0, \tau} \leqslant C_{3} \operatorname{diam}^{-m}(\tau)\left\|v_{h}\right\|_{-m, \tau}
$$

Proof. Let the affine transformation $A_{\tau}$ be as in Section 3. Set $y=A_{\tau}(x)=B_{\tau} x+$ $b_{\tau}$ and define the operator $a_{\tau}$ by $a_{\tau} v(y)=v\left(A_{\tau}^{-1}(y)\right)=v(x)$. Repeated use of the chain rule and (SA)' yields the estimates

$$
\left|D_{x}^{\alpha} \hat{v}_{h}(x)\right|^{p} \leqslant N^{|\alpha| p}\left[C_{S}^{\prime} \operatorname{diam}^{-1}(\tau)\right]^{|\alpha| p} \sum_{|\beta|=|\alpha|}\left|D_{y}^{\beta} a_{\tau} \hat{v}_{h}(y)\right|^{p}
$$

and

$$
\left|\operatorname{det} B_{\tau}^{-1}\right| \leqslant N ! C_{S}^{\prime N} \operatorname{diam}^{N}(\tau) .
$$

A change of variables then gives

$$
\left|v_{h}\right| w_{p}^{s}(\tau) \leqslant C \operatorname{diam}^{N / p-s}(\tau)\left|a_{\tau} \hat{v}_{h}\right|_{W_{p}^{s}(T)}
$$

Similarly, we obtain

$$
\left|a_{\tau} \hat{v}_{h}\right|_{W_{q}^{t}\left(c_{S}^{\prime} T\right)} \leqslant C \operatorname{diam}^{t-N / q}(\tau)\left|v_{h}\right|_{W_{q}^{t}(\tau)} .
$$

The desired estimate (4.1) now follows from the fact that, on the finite-dimensional space $P_{r-1}(T)$ and for $s$ and $t$ as considered, the seminorm $|\cdot|_{W_{p}^{s}(T)}$ is dominated by $C|\cdot|_{W_{q}^{\prime}\left(c_{S}^{\prime} T\right)}$ for some constant $C=C\left(N, r, c_{S}^{\prime}\right)$.

Corisidering the equivalence of norms on $P_{r-1}(T)$, we obtain from (4.3), with $s=0$ and $p=2$,

$$
\left\|v_{h}\right\|_{0, \tau} \leqslant C \operatorname{diam}^{N / 2}(\tau)\left\|a_{\tau} v_{h}\right\|_{-m, c_{s}^{\prime} T}
$$


From the seminorm inequality (4.3), we easily obtain, for any function $w$,

$$
\|w\|_{m, \tau} \leqslant C \operatorname{diam}^{N / 2-m}(\tau)\left\|a_{\tau} w\right\|_{m, T} .
$$

Hence,

$$
\begin{aligned}
\left\|v_{h}\right\|_{0, \tau} & \leqslant C \operatorname{diam}^{N / 2}(\tau) \sup _{a_{\tau} w \in C_{0}^{\infty}\left(c_{S}^{\prime} T\right)} \frac{\left(a_{\tau} v_{h}, a_{\tau} w\right)}{\left\|a_{\tau} w\right\|_{m, T}} \\
& \leqslant C \operatorname{diam}^{-m}(\tau) \sup _{a_{\tau} w \in C_{0}^{\infty}\left(c_{S}^{\prime} T\right)} \frac{\left(v_{h}, w\right)}{\|w\|_{m, \tau}} \\
& \leqslant C \operatorname{diam}^{-m}(\tau)\left\|v_{h}\right\|_{-m, \tau},
\end{aligned}
$$

with a constant $C$ depending only on $N, m, c_{S}^{\prime}$ and $C_{S}^{\prime}$. This shows (4.2) and completes the proof.

We shall say that $D_{h}$ is a mesh domain if $D_{h}=\operatorname{int}\left(\bigcup_{i \in M} \bar{\tau}_{i}^{h}\right)$, for some index set $M$, and we denote by $\hat{D}_{h}$ the corresponding union of simplices $\hat{\tau}_{i}^{h}$. We shall need the following local consequence of (4.1): Let $D^{\prime}$ and $D^{\prime \prime}$ be domains such that $D^{\prime} \subset D_{h}$ $\subset D^{\prime \prime} \subset D_{j}^{1}$, where $D_{h}$ is a mesh domain. Then there is a constant $C=C\left(C_{2}, C_{R}\right)$ such that

$$
\left\|v_{h}\right\|_{1, D^{\prime}} \leqslant C h_{j}^{-1}\left\|v_{h}\right\|_{0, D^{\prime \prime}}
$$

In fact, $\operatorname{since} \operatorname{diam}\left(\tau^{h}\right) \geqslant c h_{j}$ for $\tau^{h} \subset D_{j}^{1}$, we have, by (4.2),

$$
\begin{aligned}
\left\|v_{h}\right\|_{1, D_{h}}^{2} & =\sum_{\tau^{h} \subset D_{h}}\left\|v_{h}\right\|_{1, \tau^{h}}^{2} \leqslant C^{2} h_{j}^{-2} \sum_{\tau^{h} \subset D_{h}}\left\|v_{h}\right\|_{0, \tau^{h}}^{2} \\
& =C^{2} h_{j}^{-2}\left\|v_{h}\right\|_{0, D_{h}}^{2},
\end{aligned}
$$

from which (4.4) easily follows.

Together, (4.1) and (4.2) yield the following: Let $D^{\prime} \subset D_{h} \subset D^{\prime \prime}$ and assume that, for each $\tau^{h} \subset D_{h}$, one has $\operatorname{diam}\left(\tau^{h}\right) \geqslant c^{\prime} h$. Then there is a constant $C=C\left(c^{\prime}, C_{2}, C_{3}\right)$, such that

$$
\left\|v_{h}\right\|_{1, D^{\prime}} \leqslant C h^{-m-1}\left\|v_{h}\right\|_{-m, D^{\prime \prime}}
$$

To see this, we recall from Lemma 1.1 of [13] that

$$
\sum_{\tau^{h} \subset D_{h}}\left\|v_{h}\right\|_{-m, \tau}^{2} \leqslant\left\|v_{h}\right\|_{-m, D_{h}}^{2} .
$$

Hence, by (4.1) and (4.2),

$$
\begin{aligned}
\left\|v_{h}\right\|_{1, D_{h}}^{2} & =\sum_{\tau^{h} \subset D_{h}}\left\|v_{h}\right\|_{1, \tau^{h}}^{2} \leqslant C^{2} h^{-2(m+1)} \sum_{\tau^{h} \subset D_{h}}\left\|v_{h}\right\|_{-m, \tau^{h}}^{2} \\
& \leqslant C^{2} h^{-2(m+1)}\left\|v_{h}\right\|_{-m, D_{h}}^{2},
\end{aligned}
$$

which gives (4.5).

We now turn to approximation properties. The following result was proved in Eriksson [5]:

Proposition 4.2. Let $\Delta_{h}=\left\{\tau_{i}^{h}\right\}$ be a partition and $S_{h}$ an associated piecewise-polynomial space as above. Then there are constants $C_{4}$ and $C_{4}^{\prime}$ depending only on $N, \Omega, r$, 
$c_{S}^{\prime}$, and $C_{S}^{\prime}$, and, for each $\tau=\tau^{h} \in \Delta_{h}$, a neighborhood $O_{\tau}$ of $\tau$ with $\operatorname{diam}\left(O_{\tau}\right) \leqslant$ $C_{4}^{\prime} \operatorname{diam}(\tau)$, such that the following holds: For each $v \in L_{1}$ there is a $\chi_{h} \in S_{h}$ such that if $0 \leqslant l \leqslant m \leqslant r$ and $1 \leqslant p \leqslant \infty$, and if $v \in W_{p}^{m}\left(O_{\tau}\right)$, then

$$
\left\|v-\chi_{h}\right\|_{W_{p}^{\prime}(\tau)} \leqslant C_{4} \operatorname{diam}^{m-1}(\tau)\|v\|_{W_{p}^{m}\left(O_{\tau}\right)} .
$$

We have the following consequence of estimate (4.6):

Corollary 4.2. There is a constant $C$ depending only on $N, c_{S}, c_{R}, C_{R}, C_{4}$, and $C_{4}^{\prime}$ such that the following holds: Let $v \in L_{1}(\Omega)$ and let $\chi_{h}$ be given by Proposition 4.2. Furthermore, let $D^{\prime} \subset D^{\prime \prime} \subset \Omega_{j-1}$ and assume that

$$
\tau \cap D^{\prime} \neq \varnothing \quad \text { only if } O_{\tau} \subset D^{\prime \prime} .
$$

Then, for $0 \leqslant l \leqslant 1 \leqslant m \leqslant r$ and $v \in H^{m}\left(D^{\prime \prime}\right)$,

$$
\left\|v-\chi_{h}\right\|_{l, D^{\prime}} \leqslant C h_{j}^{m-l}\|v\|_{m, D^{\prime \prime}}
$$

Moreover, if $v \in W_{\infty}^{m}\left(D^{\prime \prime}\right)$, then

$$
\left\|v-\chi_{h}\right\|_{W_{\infty}^{1}\left(D^{\prime}\right)} \leqslant C h_{j}^{m-1}\|v\|_{W_{\infty}^{m}\left(D^{\prime \prime}\right)} .
$$

Proof. Let $D_{h}$ be the smallest mesh domain for which $D^{\prime} \subset D_{h}$, and note that then, for $\tau^{h} \subset D_{h}, \operatorname{diam}\left(\tau^{h}\right) \leqslant C h_{j}$ with $C=C\left(c_{R}\right)$. Since it is clear that there exists an upper bound, depending only on $N, c_{S}, c_{R}, C_{R}$, and $C_{4}^{\prime}$ for the number of $\tau$ for which $O_{\tau}$ intersects any given point, we have, by (4.6) and (4.7),

$$
\begin{aligned}
\left\|v-\chi_{h}\right\|_{l, D^{\prime}}^{2} & \leqslant \sum_{\tau^{h} \in D_{h}}\left\|v-\chi_{h}\right\|_{l, \tau^{h}}^{2} \\
& \leqslant C h_{j}^{2(m-l)} \sum_{\tau^{h} \in D_{h}}\|v\|_{m, O_{\tau} h}^{2} \leqslant C h_{j}^{2(m-l)}\|v\|_{m, D^{\prime \prime}}^{2},
\end{aligned}
$$

which proves (4.8). Estimate (4.9) is proved in the same manner.

Recall from Section 3 the definition of $J_{1}, 2^{-J_{1}}=C_{1} h^{1 /(1-\alpha)}$, which was introduced to separate the treatment of the elements closest to $x_{0}$ from that of the remaining elements of the refined mesh. We shall now fix the constant $C_{1}$ in a manner which will make (4.7) (and, hence, (4.8) and (4.9)) valid when needed in the sequel. More precisely, the proof of our crucial Lemma 6.2 below uses (4.8) and (4.9) for $j \leqslant J_{1}$ when $\operatorname{dist}\left(D^{\prime}, \Omega \backslash D^{\prime \prime}\right) \geqslant c_{1} d_{j}$. In order to justify this, we may choose

$$
C_{1}=\left(4 \cdot c_{1}^{-1} \cdot C_{4}^{\prime} \cdot c_{R}^{-1}\right)^{1 /(1-\alpha)} .
$$

It is an easy computation to show that (4.7) is then satisfied for $j \leqslant J_{1}$. If (4.7) holds, we also know that there exists a mesh domain $D_{h}$ with $D^{\prime} \subset D_{h} \subset D^{\prime \prime}$, and, hence, if $D^{\prime} \subset D^{\prime \prime} \subset D_{j}^{1}$, the inverse estimate (4.4) can be used.

Note also that Proposition 4.2 implies that $\chi_{h}$ vanishes outside a small neighborhood of $\operatorname{supp}(v)$. More precisely, if $D=\operatorname{supp}(v)$, we have

$$
\operatorname{supp}\left(\chi_{h}\right) \subset \underset{\left\{\tau: O_{\tau} \cap D \neq \varnothing\right\}}{\bigcup} \bar{\tau} .
$$

We end this section with a "superapproximation" result. Again, we refer to Eriksson [5] for the proof. 
Proposition 4.3. Let $S_{h}$ be as above, and let the constant $C_{\eta}$ be given. Then there is a constant $C=C\left(N, r, \alpha, c_{S}^{\prime}, C_{S}^{\prime}, c_{R}, C_{R}, C_{\eta}\right)$ such that the following holds: Let $D_{h}$ be a mesh domain with $D_{h} \subset D_{j}^{1}$, and let $\eta \in C^{\infty}\left(R^{N}\right)$ be constant outside $\hat{D}_{h}$ (on each component of $\left.\operatorname{int}\left(\hat{\Omega}_{h}\right) \backslash \hat{D}_{h}\right)$ and satisfy

$$
\|\eta\|_{\omega_{\infty}^{k}} \leqslant C_{\eta} d_{j}^{-k}, \quad k=0, \ldots, r .
$$

Then, given $v_{h} \in S_{h}$, there is a $\chi_{h} \in S_{h}$ such that $\operatorname{supp}\left(\eta v_{h}-\chi_{h}\right) \subset \bar{D}_{h}$ and

$$
\left\|\eta v_{h}-\chi_{h}\right\|_{1, D_{h}} \leqslant C h_{j} d_{j}^{-1}\left\|v_{h}\right\|_{1, D_{h}}+C h_{j} d_{j}^{-2}\left\|v_{h}\right\|_{0, D_{h}} \text {. }
$$

5. Main Result. High-Order Local Rate of Convergence. After the preparatory first sections, having defined the special finite-dimensional spaces $S_{h}=S_{h}\left(r, x_{0}, \alpha\right)$ and listed some of their properties, we are now ready to state and prove our main result, Theorem 5.1. The theorem states that if $u$ is a smooth function and $u_{h} \in S_{h}\left(r, x_{0}, \alpha\right)$ is its $A$-projection or, equivalently, its Galerkin approximation, then

$$
\left(u-u_{h}\right)\left(x_{0}\right)=O\left(h^{2 r-2}\right),
$$

provided $\alpha$ is sufficiently large-i.e., provided the underlying mesh is sufficiently refined close to $x_{0}$.

For a quasi-uniform mesh it is known that the pointwise convergence is of order $O\left(h^{r}\right)$ if $r>2$ (with a logarithmic modification if $r=2$ ). This was proved by Scott [19] for $N=2$ and $A(u, v)=(\nabla u, \nabla v)+(u, v)$. Our result thus shows that refining the mesh around a point $x_{0}$ considerably improves the rate of convergence of $u-u_{h}$ at this point, at least for $r>2$.

TheOREM 5.1. Let $\Omega$ and the bilinear form $A$ be as in Section 1. Furthermore, let $x_{0}$ be an arbitrary point in $\bar{\Omega}$, and for $h$ small and positive let there be given a family of partitions $\Delta_{h}\left(x_{0}, \alpha\right)$ of $\Omega$ as in Section 3. Finally, for an integer $r \geqslant 2$ let $S_{h}=$ $S_{h}\left(r, x_{0}, \alpha\right)$ be the corresponding finite-dimensional spaces as defined in Section 4. Then, for given $\alpha$ with $(r-2) /(2 r-2)<\alpha<1$, there is a constant $C$ such that, for $u \in W_{\infty}^{r}$ and $u_{h} \in S_{h}$, defined by

$$
A\left(u-u_{h}, \chi_{h}\right)=0 \quad \forall \chi_{h} \in S_{h},
$$

the estimate

$$
\left|\left(u-u_{h}\right)\left(x_{0}\right)\right| \leqslant C h^{2 r-2}\|u\|_{W_{\infty}^{r}}
$$

holds. Moreover, $C$ is independent of $x_{0}$. For $\alpha=(r-2) /(2 r-2)$, estimate (5.2) holds with $C$ replaced by $C \ln \left(h^{-1}\right)$.

Remark. Obviously, for $u \in W_{\infty}^{r}(\Omega)$, by continuous extension $u\left(x_{0}\right)$ is defined also for $x_{0} \in \partial \Omega$.

Note that Lemma 2.1 insures that $u_{h}$ is well-defined by (5.1). Note also that the very existence of a function $u_{h} \in S_{h}\left(r, x_{0}, \alpha\right)$ satisfying inequality (5.2), for $\alpha \geqslant$ $(r-2) /(2 r-2)$, is an immediate consequence of the approximation property (4.6) and the fact that $\operatorname{diam}(\tau) \leqslant C h^{1 /(1-\alpha)} \leqslant C h^{(2 r-2) / r}$ for $\tau$ close to $x_{0}$ and such $\alpha$ 's. The point of our theorem is that this high order of convergence holds for the $A$-projection $u_{h}$; i.e., that the high rate of convergence of $x_{0}$ is not destroyed by "pollution" from the coarser mesh away from $x_{0}$. 
A brief outline of the proof of Theorem 5.1 is as follows. We first write

$$
\left(u-u_{h}\right)\left(x_{0}\right)=A\left(u-u_{h}, \mathscr{G}\right)=A\left(u-\chi_{h}, \mathscr{G}-\mathscr{G}_{h}\right),
$$

where $\mathscr{G}=\mathscr{G}_{x_{0}}$ is the Green function, $\mathscr{G}_{h} \in S_{h}$ the $A^{*}$-projection of $\mathscr{G}$, and $\chi_{h}$ an arbitrary function in $S_{h}$. By the continuity of $A$, we then have to estimate $\left\|u-\chi_{h}\right\|_{W_{\infty}^{1}}$ and $\left\|\mathscr{G}-\mathscr{G}_{h}\right\|_{W_{1}^{1}}$, where, by the approximation properties of $S_{h}$, the first of these norms is bounded by $C h^{r-1}$. We thus wish to prove the same estimate for $\left\|\mathscr{G}-\mathscr{G}_{h}\right\|_{W_{1}^{1}}$. In view of the singular character of $\mathscr{G}$, this might seem too optimistic, but the idea now is that the finer mesh close to $x_{0}$ will compensate for the singularity of $\mathscr{G}$ to keep $\mathscr{G}-\mathscr{G}_{h}$ small in all of $\Omega$. To follow the above sketch of a proof, however, would require a larger lower bound for $\alpha$ than the one given in Theorem 5.1 (cf. the Corollary in [5]). In order to obtain (5.2) also for the smaller $\alpha$ 's, we shall take advantage of the fact that the $u-\chi_{h}$ part can be made smaller than $h^{r-1}$ close to the point $x_{0}$, since the mesh is refined there. Also, to deal with the singular behavior of $\mathscr{G}$ near $x_{0}$, we shall use an inverse estimate and $L_{2}$ duality to arrive at a situation where only smooth approximations of $\mathscr{G}$ have to be considered. More precisely, we have the following lemma, with the aid of which we shall then prove Theorem 5.1. For notation, see Section 3.

Lemma 5.2. Let the basic assumptions be as in Theorem 5.1. Then for given $\alpha$, with $(r-2) /(2 r-2)<\alpha<1$ and with $J_{1}=J_{1}(h, \alpha)$ as in Section 4 , there is a constant $C$ and an integer $J=J(h, \alpha) \leqslant J_{1}$ such that the following holds: Let $x_{0} \in \bar{\tau}_{0}$, and let $g \in C_{0}^{\infty}\left(\tau_{0}\right)$ be such that $\|g\|_{0}=h^{-N /(2-2 \alpha)}$. Define $G \in H^{1}$ and $G_{h} \in S_{h}=$ $S_{h}\left(r, x_{0}, \alpha\right)$ by

$$
A(v, G)=(v, g) \quad \forall v \in H^{1}, \quad A\left(\chi_{h}, G-G_{h}\right)=0 \quad \forall \chi_{h} \in S_{h} .
$$

Then there is a constant $C$ independent of $h$ such that

$$
\sum_{j \leqslant J} h_{j}^{r-1}\left\|G-G_{h}\right\|_{W_{1}^{1}\left(D_{j}\right)}+h_{J}^{r-1}\left\|G-G_{h}\right\|_{W_{1}^{1}\left(\Omega_{J+1}\right)} \leqslant C h^{2 r-2} .
$$

For $\alpha=(r-2) /(2 r-2)$, estimate (5.4) holds with $C$ replaced by $C \ln \left(h^{-1}\right)$. Moreover, $C$ is independent of $x_{0}$.

In order to understand the normalization of $g$, one may think of $g$ and $G$ as smooth approximations of the Dirac measure at $x_{0}$ and the corresponding Green function, respectively. We devote all of Section 6 to the proof of Lemma 5.2, and we proceed now to prove (5.2).

Proof of Theorem 5.1. By a sequence of standard arguments, using the triangle inequality, the inverse property (4.1) with $s=t=0, p=\infty$, and $q=2$, Hölder's inequality, and the assumption $\operatorname{diam}\left(\tau_{0}\right) \sim h^{1 /(1-\alpha)}$, we have, for any $\chi_{h} \in S_{h}$,

$$
\begin{aligned}
\left|\left(u-u_{h}\right)\left(x_{0}\right)\right| \leqslant & \left|\left(u-\chi_{h}\right)\left(x_{0}\right)\right|+\left|\left(\chi_{h}-u_{h}\right)\left(x_{0}\right)\right| \\
\leqslant & \left|\left(u-\chi_{h}\right)\left(x_{0}\right)\right|+C \operatorname{diam}^{-N / 2}\left(\tau_{0}\right)\left\|\chi_{h}-u_{h}\right\|_{L_{2}\left(\tau_{0}\right)} \\
\leqslant & \left|\left(u-\chi_{h}\right)\left(x_{0}\right)\right|+C \operatorname{diam}^{-N / 2}\left(\tau_{0}\right)\left\|u-\chi_{h}\right\|_{L_{2}\left(\tau_{0}\right)} \\
& +C \operatorname{diam}^{-N / 2}\left(\tau_{0}\right)\left\|u-u_{h}\right\|_{L_{2}\left(\tau_{0}\right)} \\
\leqslant & C\left\|u-\chi_{h}\right\|_{L_{\infty}\left(\tau_{0}\right)}+C h^{-N /(2-2 \alpha)}\left\|u-u_{h}\right\|_{L_{2}\left(\tau_{0}\right)} .
\end{aligned}
$$


By Proposition 4.2 there is a $\chi_{h} \in S_{h}$ such that

$$
\left\|u-\chi_{h}\right\|_{L_{\infty}\left(\tau_{0}\right)} \leqslant C \operatorname{diam}^{r}\left(\tau_{0}\right)\|u\|_{W_{\infty}^{r}}
$$

which, by the refinement assumption $\operatorname{diam}\left(\tau_{0}\right) \leqslant C h^{1 /(1-\alpha)}$ and the choice of $\alpha \geqslant$ $(r-2) /(2 r-2)$, can be continued to

$$
\left\|u-\chi_{h}\right\|_{L_{\infty}\left(\tau_{0}\right)} \leqslant C \operatorname{diam}^{r}\left(\tau_{0}\right)\|u\|_{W_{\infty}^{r}} \leqslant C h^{2 r-2}\|u\|_{W_{\infty}^{r}} .
$$

It now remains to estimate $\left\|u-u_{h}\right\|_{L_{2}\left(\tau_{0}\right)}$. By $L_{2}$ duality we have

$$
\left\|u-u_{h}\right\|_{L_{2}\left(\tau_{0}\right)}=\sup \left(u-u_{h}, g\right) h^{N / 2-2 \alpha)},
$$

where the sup is taken over all $g$ as in Lemma 5.2. With $G, G_{h}$, and $u_{h}$ defined by (5.3) and (5.1), we can write, for any $\chi_{h} \in S_{h}$,

$$
\left(u-u_{h}, g\right)=A\left(u-u_{h}, G\right)=A\left(u-u_{h}, G-G_{h}\right)=A\left(u-\chi_{h}, G-G_{h}\right) \text {. }
$$

Let the integer $J$ be as in Lemma 5.2. Using Corollary 4.2, we see that there is a $\chi_{h} \in S_{h}$ such that, by the continuity of $A$,

$$
\begin{aligned}
& A\left(u-\chi_{h}, G-G_{h}\right) \\
& \leqslant C\left\{\sum_{j \leqslant J}\left\|u-\chi_{h}\right\|_{W_{\infty}^{1}\left(D_{j}\right)}\left\|G-G_{h}\right\|_{W_{1}^{1}\left(D_{j}\right)}\right. \\
& \left.\quad+\left\|u-\chi_{h}\right\|_{W_{\infty}^{1}\left(\Omega_{J+1}\right)}\left\|G-G_{h}\right\|_{W_{1}^{1}\left(\Omega_{J+1}\right)}\right\} \\
& \leqslant C\|u\|_{W_{\infty}^{r}}\left\{\sum_{j \leqslant J} h_{j}^{r-1}\left\|G-G_{h}\right\|_{W_{1}^{1}\left(D_{j}\right)}+h_{J}^{r-1}\left\|G-G_{h}\right\|_{W_{1}^{1}\left(\Omega_{J+1}\right)}\right\} .
\end{aligned}
$$

The theorem now follows from (5.5) through (5.9) and by Lemma 5.2.

6. Proof of Lemma 5.2. Let us first recall from Section 3 the notation $d_{j}=2^{-j}$, $h_{j}=h d_{j}^{\alpha}, \Omega_{j}=B_{d_{j}}\left(x_{0}\right) \cap \Omega, D_{j}=\Omega_{j} \backslash \bar{\Omega}_{j+1}$, and $D_{j}^{k}=D_{j-k} \cup \cdots \cup D_{j+k}$. For given $\alpha$ with $0 \leqslant \alpha<1$, we have also defined the function $J_{1}=J_{1}(h)$ by $2^{-J_{1}}=$ $C_{1} h^{1 /(1-\alpha)}$, where the constant $C_{1}$ was chosen large enough to imply, for all $j \leqslant J_{1}$, the relative smallness of the mesh on $D_{j}$ necessary in order to derive, under the assumption $\operatorname{dist}\left(D^{\prime}, \Omega \backslash D^{\prime \prime}\right)>c_{1} d_{j}$, the local inverse estimate (4.4) and the approximation properties (4.8) and (4.9). It was also pointed out that, for $j \leqslant J_{1}$, the mesh size on $D_{j}$ is proportional to $h_{j}$ and $h_{j} d_{j}^{-1} \leqslant C$. It follows immediately from our definitions that

$$
d_{j}=2 d_{j+1} \text { and } h_{j}=2^{\alpha} h_{j+1} .
$$

This will be used frequently throughout the proofs, sometimes without explicit mention.

For the proof of Lemma 5.2, we shall need the following two preliminary lemmas. Lemma 6.1 improves the standard $H^{1}$-error estimate

$$
\left\|G-G_{h}\right\|_{1} \leqslant C h\|g\|_{0}
$$

for the special $G$ considered. Lemma 6.2 shows local $H^{1}$-estimates for $G-G_{h}$ without any specific assumptions on $G$. Proofs of these lemmas can be found in Eriksson [5]. Just take as the form in [5] the adjoint of $A$. (Cf. also the proof of Lemma 9.2 below.) 
LEMMA 6.1. For given $\alpha$, with $0 \leqslant \alpha<1$, there is a constant $C$ such that the following holds for any integer $J \leqslant J_{1}$ : Let $g \in L_{2}$ be supported in $\tau_{0}$, where $\bar{\tau}_{0}$ contains $x_{0}$. Define $G$ by

$$
A(v, G)=(v, g) \quad \forall v \in H^{1}
$$

and let $G_{h} \in S_{h}\left(r, x_{0}, \alpha\right)$ be the $A^{*}$-projection of $G$. Then, for $E=G-G_{h}$,

$$
\|E\|_{1} \leqslant C h_{J}\|g\|_{0}
$$

LEMMA 6.2. For given $\alpha$ with $0 \leqslant \alpha<1$ and $r \geqslant 2$, there is a constant $C$ such that the following holds for any integer $j \leqslant J_{1}$ : Let $G \in H^{r}\left(D_{j}^{1}\right)$, and let $G_{h} \in S_{h}=$ $S_{h}\left(r, x_{0}, \alpha\right)$ satisfy

$$
A\left(\chi_{h}, G-G_{h}\right)=0 \quad \forall \chi_{h} \in S_{h}^{0}\left(D_{j}^{1}\right) .
$$

Then, for $E=G-G_{h}$,

$$
\|E\|_{1, D_{j}} \leqslant C h_{j}^{r-1}\|G\|_{r, D_{j}^{1}}+C d_{j}^{-1}\|E\|_{0, D_{j}^{1}}
$$

Proof of Lemma 5.2. For fixed $\alpha$ with $(r-2) /(2 r-2)<\alpha<1$, we shall prove the existence of a constant $C$ and an integer $J \leqslant J_{1}$ such that

$$
\sum_{j \leqslant J} h_{j}^{r-1}\|E\|_{W_{1}^{1}\left(D_{j}\right)}+h_{J}^{r-1}\|E\|_{W_{1}^{1}\left(\Omega_{j+1}\right)} \leqslant C h^{2 r-2}
$$

where $E=G-G_{h}$ is defined as in Lemma 6.1 with the additional normalization $\|g\|_{0}=h^{-N /(2-2 \alpha)}$. By the refinement assumption $\operatorname{diam}\left(\tau_{0}\right) \sim h^{1 /(1-\alpha)}$ and the normalization of $g$, we have, by Schwarz' inequality,

$$
\|g\|_{L_{1}\left(\tau_{0}\right)} \leqslant \operatorname{diam}^{N / 2}\left(\tau_{0}\right)\|g\|_{0} \leqslant C .
$$

The choice of the integer $J \leqslant J_{1}$ will be made later on in the proof by fixing a constant $C_{*}$ (sufficiently large) and defining $J$ by the inequalities

$$
2^{-J}=d_{J} \leqslant C_{*} h^{1 /(1-\alpha)}<2 d_{J}=2^{-(J-1)} .
$$

By the definition of $h_{j}$ we then also have

$$
h_{J} \leqslant C_{*}^{\alpha} h^{1 / 1-\alpha)}<2^{\alpha} h_{J}
$$

We now set out to prove (6.6) by first estimating the term associated with $\Omega_{J+1}$. By using, in turn, Schwarz' inequality, Lemma 6.1, the normalization of $g,(6.8)$, and (6.9), we have, for $(r-2) /(2 r-2) \leqslant \alpha<1$,

$$
\begin{aligned}
h_{J}^{r-1}\|E\|_{W_{1}^{1}\left(\Omega_{J+1}\right)} & \leqslant h_{J}^{r-1} d_{J}^{N / 2}\|E\|_{1} \leqslant C h_{J}^{r} d_{J}^{N / 2}\|g\|_{0} \\
& \leqslant C h_{J}^{r} d_{J}^{N / 2} h^{-N /(2-2 \alpha)} \leqslant C C_{*}^{N / 2} h_{J}^{r} \\
& \leqslant C C_{*}^{N / 2+\alpha r} h^{r /(1-\alpha)} \leqslant C\left(C_{*}\right) h^{2 r-2},
\end{aligned}
$$

which is the desired estimate.

In order to get a similar estimate for the sum in (6.6), we first apply Schwarz' inequality to each term to obtain

$$
\sum_{j \leqslant J} h_{j}^{r-1}\|E\|_{W_{1}^{1}\left(D_{j}\right)} \leqslant C \sum_{j \leqslant J} h_{j}^{r-1} d_{j}^{N / 2}\|E\|_{1, D_{j}}
$$


We shall proceed to show, with

$$
S=\sum_{j \leqslant J} h_{j}^{r-1} d_{j}^{N / 2}\|E\|_{1, D_{i}}
$$

that, provided $C_{*}$ is chosen sufficiently large, we have

$$
S \leqslant \frac{1}{2} S+C h^{2 r-2} \text {. }
$$

This would imply $S \leqslant C h^{2 r-2}$ and, thus, together with (6.11) and (6.10), show the desired estimate (6.6). To complete the proof of Lemma 5.2, we shall also prove that (6.12) holds for $\alpha=(r-2) /(2 r-2)$ if $C$ is replaced by $C \ln \left(h^{-1}\right)$. Moreover, as is easily seen by tracking constants, we shall prove (6.6) with $C$ depending only on $N, \Omega, A, \alpha, r$, and given constants. That is, the final claim of Lemma 5.2 will be fulfilled.

In order to show (6.12) we first split the sum $S$ and then make use of Lemma 6.2. We have

$$
\begin{aligned}
S= & \sum_{j=J-2}^{J}+\sum_{j \leqslant J-3}=S_{1}+\sum_{j \leqslant J-3} h_{j}^{r-1} d_{j}^{N / 2}\|E\|_{1, D_{j}} \\
\leqslant & S_{1}+C \sum_{j \leqslant J-3} h_{j}^{2 r-2} d_{j}^{N / 2}\|G\|_{r, D_{j}^{1}} \\
& +C \sum_{j \leqslant J-3} h_{j}^{r-1} d_{j}^{N / 2-1}\|E\|_{0, D_{j}^{1}} \\
\leqslant & S_{1}+S_{2}+C_{5} \sum_{j \leqslant J-2} h_{j}^{r-1} d_{j}^{N / 2-1}\|E\|_{0, D_{i}},
\end{aligned}
$$

where we have also used (6.1) in the last step.

The sum $S_{1}$, only consisting of three terms, has essentially already been estimated. In fact, by (6.1) and part of (6.10), we have

$$
S_{1} \leqslant 3 h_{J-2}^{r-1} d_{J-2}^{N / 2}\|E\|_{1} \leqslant C h_{J}^{r-1} d_{J}^{N / 2}\|E\|_{1} \leqslant C\left(C_{*}\right) h^{2 r-2} .
$$

For the estimation of $S_{2}$ we first note that, since $D_{j}$ and $\operatorname{supp}(g)$ are separated by a distance of order $d_{j}$, we have, from Lemma 2.3,

$$
\|G\|_{W_{\infty}^{r}\left(D_{j}\right)} \leqslant C d_{j}^{-N+2-r}\|g\|_{L_{1}\left(\tau_{0}\right)}
$$

By Hölder's inequality and (6.7), this shows

$$
\|G\|_{r, D_{j}} \leqslant C d_{j}^{N / 2}\|G\|_{W_{\infty}^{r}\left(D_{j}\right)} \leqslant C d_{j}^{-N / 2+2-r} .
$$

Let $j_{1}$ be the smallest integer for which $D_{j} \neq \varnothing$. Using (6.15) we obtain

$$
\begin{aligned}
S_{2} & \leqslant C \sum_{j \leqslant J-2} h_{j}^{2 r-2} d_{j}^{N / 2}\|G\|_{r, D_{j}} \leqslant C \sum_{j=j_{1}}^{J} h_{j}^{2 r-2} d_{j}^{2-r} \\
& \leqslant C h^{2 r-2} \sum_{j=j_{1}}^{J} d_{j}^{\alpha(2 r-2)+2-r} \leqslant C h^{2 r-2},
\end{aligned}
$$

where we have used that $\alpha>(r-2) /(2 r-2)$ in the last step. In the case $\alpha=$ $(r-2) /(2 r-2)$, all terms in the sum equal 1 , and so, since by $(6.8)$ the number of 
terms is proportional to $\ln \left(h^{-1}\right)$, we have

$$
S_{2} \leqslant C \ln \left(h^{-1}\right) h^{2 r-2} .
$$

It now remains to show that

$$
C_{5} \sum_{j \leqslant J-2} h_{j}^{r-1} d_{j}^{N / 2-1}\|E\|_{0, D_{j}} \leqslant \frac{1}{2} S+C h^{2 r-2} .
$$

For this we first write, by duality,

$$
\|E\|_{0, D_{j}}=\sup \left(f_{j}, E\right)
$$

where the supremum is taken over all functions $f_{j}$ supported in $D_{j}$ and of unit $L_{2}$-norm. For such $f_{j}$ we define $F_{j}$ by

$$
A\left(F_{j}, v\right)=\left(f_{j}, v\right) \quad \forall v \in H^{1}
$$

and note that new applications of Hölder's inequality and Lemma 2.3, together with the normalization of $f_{j}$, give, for $i \neq j-1, j, j+1$,

$$
\begin{aligned}
\left\|F_{j}\right\|_{r, D_{i}} & \leqslant C d_{i}^{N / 2}\left\|F_{j}\right\|_{W_{\infty}^{r}\left(D_{i}\right)} \leqslant C d_{i}^{N / 2} d_{j}^{-N+2-r}\left\|f_{j}\right\|_{L_{1}\left(D_{j}\right)} \\
& \leqslant C d_{i}^{N / 2} d_{j}^{-N / 2+2-r} .
\end{aligned}
$$

By (6.19), the definition of $E$, and the $H^{1}$-continuity of $A$, we have, for arbitrary $\chi_{h} \in S_{h}$,

$$
\begin{aligned}
\left(f_{j}, E\right)= & A\left(F_{j}, E\right)=A\left(F_{j}-\chi_{h}, E\right) \\
\leqslant & C\left\|F_{j}-\chi_{h}\right\|_{1, D_{j}^{2}}\|E\|_{1, D_{j}^{2}}+C \sum_{\substack{i \leqslant J \\
i \neq j-2, \ldots, j+2}}\left\|F_{j}-\chi_{h}\right\|_{1, D_{i}}\|E\|_{1, D_{i}} \\
& +C\|E\|_{1, \Omega_{j+1}}\left\|F_{j}-\chi_{h}\right\|_{1, \Omega_{J+1}} \\
= & I_{1}+I_{2}+I_{3} .
\end{aligned}
$$

We next intend to estimate $I_{1}, I_{2}$, and $I_{3}$ separately, with $\chi_{h}$ approximating $F_{j}$ as in Proposition 4.2.

By the approximation property (4.8), elliptic $H^{2}$-regularity, and the normalization of $f_{j}$, we first have, for $I_{1}$,

$$
I_{1} \leqslant C\|E\|_{1, D_{j}^{2}} h_{j}\left\|F_{j}\right\|_{2} \leqslant C\|E\|_{1, D_{j}^{2}} h_{j}\left\|f_{j}\right\|_{0} \leqslant C h_{j}\|E\|_{1, D_{j}^{2}} .
$$

We next use (4.8) and (6.20) to estimate $I_{2}$. We have

$$
\begin{aligned}
I_{2} & \leqslant C \sum_{\substack{i \leqslant J \\
i \neq j-2, \ldots, j+2}}\|E\|_{1, D_{i}} h_{i}^{r-1}\left\|F_{j}\right\|_{r, D_{i}^{1}} \\
& \leqslant C \sum_{\substack{i \leqslant J \\
i \neq j-2, \ldots, j+2}}\|E\|_{1, D_{i}} h_{i}^{r-1} d_{i}^{N / 2} d_{j}^{-N / 2+2-r} \\
& \leqslant C d_{j}^{-N / 2+2-r} \sum_{i \leqslant J} h_{i}^{r-1} d_{i}^{N / 2}\|E\|_{1, D_{i}}=C d_{j}^{-N / 2+2-r} S .
\end{aligned}
$$

It remains to estimate $I_{3}$. Since we shall only consider $F_{j}$ with $j \leqslant J-2$, i.e., with $\operatorname{supp}\left(f_{j}\right)$ separated from $\Omega_{J}$, we use Lemma 2.3 to derive

$$
\left\|F_{j}\right\|_{r, \Omega_{J}} \leqslant C d_{J}^{N / 2} d_{j}^{-N / 2+2-r} .
$$


From this, the approximation property (4.8), and again using part of (6.10), we have

$$
\begin{aligned}
I_{3} & \leqslant C\|E\|_{1, \Omega_{J+1}} h_{J}^{r-1}\left\|F_{j}\right\|_{r, \Omega_{J}} \\
& \leqslant C\|E\|_{1, \Omega_{J+1}} h_{J}^{r-1} d_{J}^{N / 2} d_{j}^{-N / 2+2-r} \\
& \leqslant C d_{j}^{-N / 2+2-r} h_{J}^{r-1} d_{J}^{N / 2}\|E\|_{1} \leqslant C\left(C_{*}\right) h^{2 r-2} d_{j}^{-N / 2+2-r} .
\end{aligned}
$$

Collecting (6.18), (6.21), and the above estimates for $I_{1}, I_{2}$, and $I_{3}$, we have shown

$$
\|E\|_{0, D_{j}} \leqslant C h_{j}\|E\|_{1, D_{j}^{2}}+C d_{j}^{-N / 2+2-r} S+C\left(C_{*}\right) h^{2 r-2} d_{j}^{-N / 2+2-r} .
$$

Estimate (6.22) inserted into the sum in (6.17) gives

$$
\begin{aligned}
C_{5} & \sum_{j \leqslant J-2} h_{j}^{r-1} d_{j}^{N / 2-1}\|E\|_{0, D_{j}} \\
\leqslant & C \sum_{j \leqslant J}\left(h_{j} d_{j}^{-1}\right) h_{j}^{r-1} d_{j}^{N / 2}\|E\|_{1, D_{j}} \\
& +C S \sum_{j \leqslant J} h_{j}^{r-1} d_{j}^{1-r}+C\left(C_{*}\right) h^{2 r-2} \sum_{j \leqslant J} h_{j}^{r-1} d_{j}^{1-r} \\
& \leqslant C_{6}\left(\max _{j \leqslant J}\left(h_{j} d_{j}^{-1}\right)+\sum_{j \leqslant J} h_{j}^{r-1} d_{j}^{1-r}\right) S+C\left(C_{*}\right) h^{2 r-2} \sum_{j \leqslant J} h_{j}^{r-1} d_{j}^{1-r},
\end{aligned}
$$

where the constant $C_{6}$ is independent of the choice of $C_{*}$. As is easily seen from (6.23), all that remains in order to obtain (6.17) is to show that, for a suitable choice of $C_{*}$, we have

$$
C_{6}\left(\max _{j \leqslant J}\left(h_{j} d_{j}^{-1}\right)+\sum_{j \leqslant J} h_{j}^{r-1} d_{j}^{1-r}\right) \leqslant \frac{1}{2} .
$$

By the definition of $h_{j}$, the assumption $\alpha<1,(6.8)$, and for $C_{*}$ sufficiently large, we have

$$
\max _{j \leqslant J}\left(h_{j} d_{j}^{-1}\right)=\max _{j \leqslant J}\left(h d_{j}^{\alpha-1}\right)=h d_{J}^{\alpha-1}<\frac{2^{1-\alpha}}{C_{*}^{1-\alpha}} \leqslant \frac{1}{4 C_{6}} .
$$

Since $\alpha<1$ and $r \geqslant 2$, we have, again for $C_{*}$ sufficiently large,

$$
\sum_{j \leqslant J} h_{j}^{r-1} d_{j}^{1-r}=h^{r-1} \sum_{j \leqslant J} d_{j}^{(\alpha-1)(r-1)}=h^{r-1} d_{J}^{(\alpha-1)(r-1)}\left(1-2^{(\alpha-1)(r-1)}\right)^{-1}
$$

$$
<\frac{2^{(1-\alpha)(r-1)}\left(1-2^{(\alpha-1)(r-1)}\right)^{-1}}{C_{*}^{(1-\alpha)(r-1)}} \leqslant \frac{1}{4 C_{6}},
$$

where we have again used the right inequality of (6.8). Now fixing the constant $C_{*}$ so that both (i) and (ii) hold, we have obtained the desired inequality (6.24) and, thereby, finally proved Lemma 5.2.

7. High-Order Local Convergence for Derivatives. In this section we shall prove that, for $\alpha$ sufficiently large, we have $O\left(h^{2 r-2}\right)$ convergence at $x_{0}$ also for the first-order derivatives. More precisely, we have the following result:

TheOREM 7.1. Let $\Omega$ and the bilinear form $A$ be as in Section 1. Furthermore, let $x_{0}$ be an arbitrary point in $\bar{\Omega}$, let $r \geqslant 2$ be an integer, and for $h$ small and positive let there be given a family of partitions $\Delta_{h}\left(x_{0}, \alpha\right)$ of $\Omega$ and the corresponding finite-dimensional 
spaces $S_{h}=S_{h}\left(r, x_{0}, \alpha\right)$ as in Sections 3 and 4. Then, for given $\alpha$ with $1 / 2<\alpha<1$, there is a constant $C$ such that, for $u \in W_{\infty}^{r}$ and $u_{h} \in S_{h}$, the A-projection of $u$, the estimate

$$
\left|\nabla\left(u-u_{h}\right)\left(x_{0}\right)\right| \leqslant C h^{2 r-2}\|u\|_{W_{\infty}^{r}}
$$

holds. If $\alpha=1 / 2$, estimate (7.1) holds with $C$ replaced by $C \ln \left(h^{-1}\right)$. Moreover, the constant $C$ is independent of $x_{0}$.

For the proof we shall need the following technical result:

LEMMA 7.2. Let the element $\tau$, the simplex $\hat{\tau}$, and the affine transformation $A_{\tau}$ satisfy assumption (SA)' of Section 3, and let $r \geqslant 2$ be an integer. Then there is a constant $C=C\left(N, r, c_{S}^{\prime}, C_{S}^{\prime}\right)$ such that, for any $p \in P_{r}(\hat{\tau})$, we have

$$
\|p\|_{L_{\infty}(\tau)} \leqslant C \sup (p, g)
$$

where the supremum is taken over all $g \in C_{0}^{\infty}(\tau)$ with

$$
\operatorname{diam}^{N / 2}(\tau)\|g\|_{0}+\operatorname{diam}^{(N+2) / 2}(\tau)\|g\|_{1} \leqslant 1
$$

Proof. Define, as before, the operator $a_{\tau}$ by $a_{\tau} v(y)=v\left(A_{\tau}^{-1}(y)\right)$. Then

$$
\|p\|_{L_{\infty}(\tau)} \leqslant\|p\|_{L_{\infty}(\hat{\tau})}=\left\|a_{\tau} p\right\|_{L_{\infty}(T)} .
$$

With $c_{S}^{\prime}$ given in assumption (SA), let $B_{2 c^{\prime}}\left(y^{\prime}\right)$ be the largest ball contained in $c_{S}^{\prime} T$, and set $B=B_{c^{\prime}}\left(y^{\prime}\right)$. By the equivalence of norms on $P_{r}(T)$, there is a constant $C=C\left(N, r, c_{S}^{\prime}\right)$ such that

$$
\left\|p^{\prime}\right\|_{L_{\infty}(T)} \leqslant C\left\|p^{\prime}\right\|_{L_{x}(B)} \quad \forall p^{\prime} \in P_{r} .
$$

Let $M$ be a fixed function in $C_{0}^{\infty}\left(B_{1}(0)\right)$ satisfying

$$
\left(p^{\prime}, M\right)=p^{\prime}(0) \quad \forall p^{\prime} \in P_{r}
$$

(cf. Hilbert [8]) and set $M_{z}(y)=c^{\prime-N} M\left((y-z) / c^{\prime}\right)$. Then

$$
\left(p^{\prime}, M_{z}\right)=p^{\prime}(z) \quad \forall p^{\prime} \in P_{r},
$$

and, hence,

$$
\left\|p^{\prime}\right\|_{L_{\infty}(B)}=\sup _{z \in B}\left(p^{\prime}, M_{z}\right) \quad \forall p^{\prime} \in P_{r} .
$$

Noting that $a_{\tau} p \in P_{r}$, we now obtain

$$
\|p\|_{L_{\infty}(\tau)} \leqslant C \sup _{z \in B}\left(a_{\tau} p, M_{z}\right)=C \sup _{z \in B}\left(p,\left|\operatorname{det} B_{\tau}\right| a_{\tau}^{-1} M_{z}\right)
$$

where we have changed variables in the last step. To prove (7.2) it is then sufficient to show that, for some (small) constant $\delta$, the functions $g=\delta\left|\operatorname{det} B_{\tau}\right| a_{\tau}^{-1} M_{z}$, for $z \in B$, satisfy $g \in C_{0}^{\infty}(\tau)$ and (7.2). However, since $M \in C_{0}^{\infty}\left(B_{1}(0)\right)$, it follows from the definitions of $B, M_{z}$, and $a_{\tau}^{-1}$ and from (SA)' that

$$
g=\delta\left|\operatorname{det} B_{\tau}\right| a_{\tau}^{-1} M_{z} \in C_{0}^{\infty}(\tau) .
$$

Moreover, since $M$ is bounded, so is $a_{\tau}^{-1} M_{z}$, and hence by Hölder's inequality and for suitable $\delta$ 's,

$$
\operatorname{diam}^{N / 2}(\tau)\|g\|_{0, \tau} \leqslant \operatorname{diam}^{N}(\tau)\|g\|_{L_{\infty}} \leqslant \delta \operatorname{diam}^{N}(\tau)\left|\operatorname{det} B_{\tau}\right|\left\|a_{\tau}^{-1} M_{z}\right\|_{L_{x}} \leqslant 1 / 2 .
$$


Similarly,

which completes the proof.

$$
\begin{aligned}
\operatorname{diam}^{(N+2) / 2}(\tau)\|g\|_{1, \tau} & \leqslant \delta C \operatorname{diam}^{N+1}(\tau)\left|\operatorname{det} B_{\tau}\right|\left\|a_{\tau}^{-1} M_{z}\right\|_{W_{\infty}^{1}} \\
& \leqslant C \delta \operatorname{diam}(\tau)\left\|a_{\tau}^{-1} M_{z}\right\|_{W_{\infty}^{1}} \leqslant 1 / 2,
\end{aligned}
$$

Proof of Theorem 7.1. We shall prove (7.1) with $\nabla$ replaced by any $\partial_{j}=\partial / \partial x_{j}$. Let $x_{0} \in \bar{\tau}_{0}$. We shall use Lemma 7.2 with $\tau=\tau_{0}$ and $p=\partial_{j}\left(\chi_{h}-u_{h}\right) \in P_{r-1}\left(\hat{\tau}_{0}\right)$. Using the triangle inequality, Lemma 7.2, and Hölder's inequality, we then have, for any $\chi_{h} \in S_{h}$,

$$
\begin{aligned}
\left|\left(\partial_{j} u-\partial_{j} u_{h}\right)\left(x_{0}\right)\right| \leqslant & \left|\left(\partial_{j} u-\partial_{j} \chi_{h}\right)\left(x_{0}\right)\right|+\left|\left(\partial_{j} \chi_{h}-\partial_{j} u_{h}\right)\left(x_{0}\right)\right| \\
\leqslant & \left|\left(\partial_{j} u-\partial_{j} \chi_{h}\right)\left(x_{0}\right)\right|+C \sup \left(\partial_{j} \chi_{h}-\partial_{j} u_{h}, g\right) \\
\leqslant & \left\|\partial_{j} u-\partial_{j} \chi_{h}\right\|_{L_{\infty}\left(\tau_{0}\right)}+C \sup \left(\partial_{j} u-\partial_{j} \chi_{h}, g\right) \\
& +C \sup \left(\partial_{j} u-\partial_{j} u_{h}, g\right) \\
\leqslant & \left\|\partial_{j} u-\partial_{j} \chi_{h}\right\|_{L_{\infty}\left(\tau_{0}\right)}+C \sup \left\|\partial_{j} u-\partial_{j} \chi_{h}\right\|_{L_{\infty}\left(\tau_{0}\right)}\|g\|_{L_{1}\left(\tau_{0}\right)} \\
& +C \sup \left(\partial_{j} u-\partial_{j} u_{h}, g\right),
\end{aligned}
$$

where the supremum is taken over all $g \in C_{0}^{\infty}\left(\tau_{0}\right)$ with

$$
\operatorname{diam}^{N / 2}\left(\tau_{0}\right)\|g\|_{0}+\operatorname{diam}^{(N+2) / 2}\left(\tau_{0}\right)\|g\|_{1} \leqslant 1 .
$$

Since, for $\alpha \geqslant 1 / 2$, the refinement assumption (RA) implies

$$
\operatorname{diam}\left(\tau_{0}\right) \leqslant C h^{1 /(1-\alpha)} \leqslant C h^{2},
$$

Proposition 4.2 yields a $\chi_{h} \in S_{h}$ such that

$$
\left\|\partial_{j} u-\partial_{j} \chi_{h}\right\|_{L_{\infty}\left(\tau_{0}\right)} \leqslant C \operatorname{diam}^{r-1}\left(\tau_{0}\right)\|u\|_{W_{\infty}^{r}} \leqslant C h^{2 r-2}\|u\|_{W_{\infty}^{r}} .
$$

Moreover, by Schwarz' inequality and the normalization of $g$, we have

$$
\|g\|_{L_{1}\left(\tau_{0}\right)} \leqslant \operatorname{diam}^{N / 2}\left(\tau_{0}\right)\|g\|_{0} \leqslant 1
$$

and, hence, the first two terms on the last line of (7.3) can be estimated in the desired way. In the last term we first integrate by parts. We then put $g^{\prime}=-\partial_{j} g$ and define $G^{\prime} \in H^{1}$ and $G_{h}^{\prime} \in S_{h}$ by

$$
A\left(v, G^{\prime}\right)=\left(v, g^{\prime}\right) \quad \forall v \in H^{1}, \quad A\left(\chi_{h}, G^{\prime}-G_{h}^{\prime}\right)=0 \quad \forall \chi_{h} \in S_{h} .
$$

Following the proof of Theorem 5.1 with $g, G$, and $G_{h}$ replaced by $g^{\prime}, G^{\prime}$, and $G_{h}^{\prime}$, we then obtain

$$
\begin{aligned}
& \left(\partial_{j} u-\partial_{j} u_{h}, g\right)=\left(u-u_{h},-\partial_{j} g\right)=\left(u-u_{h}, g^{\prime}\right)=A\left(u-u_{h}, G^{\prime}\right) \\
& =A\left(u-u_{h}, G^{\prime}-G_{h}^{\prime}\right)=A\left(u-\chi_{h}, G^{\prime}-G_{h}^{\prime}\right) \\
& \leqslant C\left\{\sum_{j \leqslant J}\left\|u-\chi_{h}\right\|_{W_{\infty}^{1}\left(D_{j}\right)}\left\|G^{\prime}-G_{h}^{\prime}\right\| W_{1}^{1}\left(D_{j}\right)\right. \\
& \left.+\left\|u-\chi_{h}\right\|_{W_{\infty}^{1}\left(\Omega_{J+1}\right)}\left\|G^{\prime}-G_{h}^{\prime}\right\|_{W_{1}^{1}\left(\Omega_{J+1}\right)}\right\} \\
& \leqslant C\|u\|_{W_{\infty}^{r}}\left\{\sum_{j \leqslant J} h_{j}^{r-1}\left\|G^{\prime}-G_{h}^{\prime}\right\|_{W_{1}^{1}\left(D_{j}\right)}+h_{J}^{r-1}\left\|G^{\prime}-G_{h}^{\prime}\right\|_{W_{1}^{1}\left(\Omega_{J+1}\right)}\right\}
\end{aligned}
$$


The desired estimate now follows from (7.3)-(7.7) and by Lemma 7.3 below. The independence of $x_{0}$ of the constant $C$ is easily checked. This completes the proof of Theorem 7.1.

LeMma 7.3. Let the basic assumptions be as in Theorem 7.1. Then for given $\alpha$, with $1 / 2<\alpha<1$ and $J_{1}$ as before, there is a constant $C$ and an integer $J \leqslant J_{1}$ such that the following holds: Let $x_{0} \in \bar{\tau}_{0}$ and let $g \in C_{0}^{\infty}\left(\tau_{0}\right)$ satisfy

$$
\operatorname{diam}^{N / 2}\left(\tau_{0}\right)\|g\|_{0}+\operatorname{diam}^{(N+2) / 2}\left(\tau_{0}\right)\|g\|_{1} \leqslant 1 .
$$

Set $g^{\prime}=-\partial_{j} g$ and define $G^{\prime} \in H^{1}$ and $G_{h}^{\prime} \in S_{h}$ as in (7.6). Then for $E^{\prime}=G^{\prime}-G_{h}^{\prime}$,

$$
\sum_{j \leqslant J} h_{j}^{r-1}\left\|E^{\prime}\right\|_{W_{1}^{1}\left(D_{j}\right)}+h_{J}^{r-1}\left\|E^{\prime}\right\|_{W_{1}^{1}\left(\Omega_{J+1}\right)} \leqslant C h^{2 r-2} \text {. }
$$

If $\alpha=1 / 2$, estimate (7.8) holds with $C$ replaced by $C \ln \left(h^{-1}\right)$.

Proof. We first estimate the term associated with $\Omega_{J+1}$. As in the proof of Lemma 5.2 , we shall choose $J \leqslant J_{1}$ such that

$$
d_{J} \leqslant C_{*} h^{1 /(1-\alpha)}<2 d_{J} \text { and } h_{J} \leqslant C_{*}^{\alpha} h^{1 /(1-\alpha)}<2^{\alpha} h_{J},
$$

where the constant $C_{*}$ will be determined later in the proof. Then, by the refinement assumption, $h_{J}, d_{J}, h^{1 /(1-\alpha)}$, and $\operatorname{diam}\left(\tau_{0}\right)$ are all of the same order. Hence, using Schwarz' inequality, Lemma 6.1, and the bound for $g$, we have, for $\alpha \geqslant 1 / 2$,

$$
\begin{aligned}
h_{J}^{r-1}\left\|E^{\prime}\right\|_{W_{1}^{1}\left(\Omega_{J+1}\right)} & \leqslant h_{J}^{r-1} d_{J}^{N / 2}\left\|E^{\prime}\right\|_{1} \leqslant C h_{J}^{r-1} d_{J}^{N / 2} h_{J}\left\|g^{\prime}\right\|_{0} \\
& \leqslant C\left(C_{*}\right) h^{(r-1) /(1-\alpha)} \operatorname{diam}^{(N+2) / 2}\left(\tau_{0}\right)\|g\|_{1} \leqslant C\left(C_{*}\right) h^{2 r-2},
\end{aligned}
$$

which shows the desired estimate for this term.

We now proceed to estimate the sum in (7.8). Setting

$$
S=\sum_{j \leqslant J} h_{j}^{r-1} d_{j}^{N / 2}\left\|E^{\prime}\right\|_{1, D_{j}}
$$

we have, by Schwarz' inequality,

$$
\sum_{j \leqslant J} h_{j}^{r-1}\left\|E^{\prime}\right\|_{W_{1}^{1}\left(D_{j}\right)} \leqslant C S
$$

We shall first show that

$$
S \leqslant C h^{2 r-2}+C \sum_{j \leqslant J-2} h_{j}^{2 r-2} d_{j}^{N / 2}\left\|G^{\prime}\right\|_{r, D_{j}} .
$$

We shall again use the obvious equalities (6.1), $d_{j}=2 d_{j+1}$, and $h_{j}=2^{\alpha} h_{j+1}$. In order to estimate the three last terms of $S$, we shall use part of (7.9), and to the rest of the terms we apply Lemma 6.2. We then have

$$
\begin{aligned}
S= & \sum_{j=J-2}^{J}+\sum_{j \leqslant J-3} \leqslant 3 h_{J-2}^{r-1} d_{J-2}^{N / 2}\left\|E^{\prime}\right\|_{1}+\sum_{j \leqslant J-3} h_{j}^{r-1} d_{j}^{N / 2}\left\|E^{\prime}\right\|_{1, D_{j}} \\
\leqslant & C h_{J}^{r-1} d_{J}^{N / 2}\left\|E^{\prime}\right\|_{1}+C \sum_{j \leqslant J-3} h_{j}^{2 r-2} d_{j}^{N / 2}\left\|G^{\prime}\right\|_{r, D_{j}^{1}} \\
& +C \sum_{j \leqslant J-3} h_{j}^{r-1} d_{j}^{N / 2-1}\left\|E^{\prime}\right\|_{0, D_{j}^{1}} \\
\leqslant & C\left(C_{*}\right) h^{2 r-2}+C \sum_{j \leqslant J-2} h_{j}^{2 r-2} d_{j}^{N / 2}\left\|G^{\prime}\right\|_{r, D_{j}} \\
& +C_{5}^{\prime} \sum_{j \leqslant J-2} h_{j}^{r-1} d_{j}^{N / 2-1}\left\|E^{\prime}\right\|_{0, D_{j}} .
\end{aligned}
$$


In order to estimate the last sum, we proceed, as in Section 6, by first estimating $\left\|E^{\prime}\right\|_{0, D_{j}}$. Following (6.18)-(6.21), we obtain three terms, $I_{1}^{\prime}, I_{2}^{\prime}$, and $I_{3}^{\prime}$, which have to be estimated. The terms $I_{1}^{\prime}$ and $I_{2}^{\prime}$ are bounded exactly as in Section 6 but for the additional "primes". In order to bound $I_{3}^{\prime}$ we use part of (7.9) instead of (6.10). As in Section 6 , if $C_{*}$ is sufficiently large, we finally arrive at the estimate

$$
C_{5}^{\prime} \sum_{j \leqslant J-2} h_{j}^{r-1} d_{j}^{N / 2-1}\left\|E^{\prime}\right\|_{0, D} \leqslant C\left(C_{*}\right) h^{2 r-2}+\frac{1}{2} S .
$$

By a simple kickback argument, (7.11) follows from (7.12) and (7.13). It now remains to show

$$
\sum_{j \leqslant J-2} h_{j}^{2 r-2} d_{j}^{N / 2}\left\|G^{\prime}\right\|_{r, D_{j}} \leqslant C h^{2 r-2} .
$$

Using the representation (2.5) we have, for $G^{\prime}$,

$$
G^{\prime}(x)=\int_{\Omega} \mathscr{G}(x, y) g^{\prime}(y) d y=-\int_{\Omega} \mathscr{G}(x, y) \partial_{j} g(y) d y .
$$

Differentiation under the integral sign and integration by parts give

$$
D^{\beta} G^{\prime}(x)=\int_{\Omega} \partial_{j} D_{x}^{\beta} \mathscr{G}(x, y) g(y) d y .
$$

For $j \leqslant J_{1}$ and any $x \in D_{j}$ we have that $\operatorname{dist}(x, \operatorname{supp}(g)) \geqslant c d_{j}$. Thus, Hölder's inequality and estimate (2.6) yield

$$
\left|D^{\beta} G^{\prime}(x)\right| \leqslant C d_{j}^{-N+2-|\beta|-1}\|g\|_{L_{1}\left(\tau_{0}\right)},
$$

and, hence,

$$
\left\|G^{\prime}\right\|_{\left.W_{\infty}^{r}(D)\right)} \leqslant C d_{j}^{-N+1-r}\|g\|_{L_{1}\left(\tau_{0}\right)} .
$$

By Schwarz' inequality and the previously obtained estimate $\|g\|_{L_{1}\left(\tau_{0}\right)} \leqslant C$, we have

$$
\left\|G^{\prime}\right\|_{r, D,} \leqslant C d_{j}^{-N / 2+1-r} \text {. }
$$

Inserted in (7.14), with $j_{1}$ as before, for fixed $\alpha>1 / 2$, this gives

$$
\begin{gathered}
\sum_{j \leqslant J-2} h_{j}^{2 r-2} d_{j}^{N / 2}\left\|G^{\prime}\right\|_{r . D_{j}} \leqslant C \sum_{j=j_{1}}^{J} h_{j}^{2 r-2} d_{j}^{1-r} \\
=C h^{2 r-2} \sum_{j=j_{1}}^{J} d_{j}^{\alpha(2 r-2)+1-r} \leqslant C h^{2 r-2} .
\end{gathered}
$$

This shows (7.14). In order to complete the proof of Lemma 7.3, we note that in the case $\alpha=1 / 2$ all terms in the last sum equal 1. Estimating the number of terms, we obtain the final bound $C \ln \left(h^{-1}\right) h^{2 r-2}$.

8. A Negative-Norm Estimate. In this section we shall prove a negative-norm estimate referred to in Sections 9 and 10. The result is well-known from, e.g., Bramble and Osborn [3], but will be proved here for completeness. However, we shall first show the following result on approximation properties of the spaces $S_{h}\left(\Omega^{\prime}\right)$. 
Proposition 8.1. Let the domain $\Omega$, the bilinear form $A$, and the family of finite-dimensional spaces $S_{h}=S_{h}\left(r, x_{0}, \alpha\right)$ be as before. Moreover, let $\Omega^{\prime}$ be a subdomain on $\Omega$ with $C^{\infty}$ boundary $\partial \Omega^{\prime}$. Then there is a constant $C$, and for each given $v \in L_{1}\left(\Omega^{\prime}\right)$ a $\chi_{h} \in S_{h}\left(\Omega^{\prime}\right)$ such that the following holds: If $j \leqslant J_{1}, D^{\prime} \subset D^{\prime \prime} \subset\left(\Omega^{\prime} \cap\right.$ $\left.B_{j-1}\left(x_{0}\right)\right), \operatorname{dist}\left(D^{\prime}, \Omega^{\prime} \backslash D^{\prime \prime}\right) \geqslant c_{1} d_{j}, 1 \leqslant p \leqslant \infty, 0 \leqslant l \leqslant 1 \leqslant m \leqslant r$, and if $v \in$ $W_{p}^{m}\left(D^{\prime \prime}\right)$, then

$$
\left\|v-\chi_{h}\right\|_{W_{p}^{\prime}\left(D^{\prime}\right)} \leqslant C h_{j}^{m-l}\|v\|_{W_{p}^{m}\left(D^{\prime \prime}\right)} .
$$

In particular, if $v \in H^{r}\left(\Omega^{\prime}\right)$, then

$$
\left\|v-\chi_{h}\right\|_{1, \Omega^{\prime}} \leqslant C h^{r-1}\|v\|_{r, \Omega^{\prime}}
$$

Proof. There exist (cf. Eriksson [5]) an extension operator $E: L_{1}\left(\Omega^{\prime}\right) \rightarrow L_{1}\left(R^{N}\right)$ and constants $C$ and $c_{E}$ such that, for $x \in \bar{\Omega}^{\prime}$,

$$
\|E v\|_{W_{p}^{m}\left(B_{d}(x)\right)} \leqslant C\|v\|_{W_{p}^{m}\left(B_{c E^{d}}(x) \cap \Omega^{\prime}\right)} .
$$

Let $\chi_{h}$ approximate $E v$ as in Proposition 4.2. Let $\tau^{\prime}$ be an element of the partition of $\Omega^{\prime}$ induced by $\Delta_{h}$. Following the proof of Proposition 4.2 given in [5], we obtain the estimate

$$
\left\|v-\chi_{h}\right\|_{W_{p}^{\prime}\left(\tau^{\prime}\right)} \leqslant C \operatorname{diam}^{m-l}(\tau)\|v\|_{W_{p}^{m}\left(O_{\tau^{\prime}}\right)},
$$

where $\tau^{\prime} \subset \tau \in \Delta_{h}$, and where $O_{\tau^{\prime}}$ is a neighborhood of $\tau^{\prime}$ in $\Omega^{\prime}$ having diameter of order $h_{j}$. Note also that, by the choice of $J_{1}$, we can assume that condition (4.7) holds for $O_{\tau^{\prime}}$. But then estimates (8.1) and (8.2) are easily derived from (8.3) by the same means as we earlier obtained (4.8) and (4.9).

Proposition 8.1 will be used in Sections 9 and 10. In particular, (8.2) implies the desired negative-norm estimate:

THEOREM 8.2. Let the domain $\Omega$, the bilinear form $A$, and the family of finite-dimensional spaces $S_{h}$ be as before. Moreover, let $\Omega^{\prime}$ be a subdomain of $\Omega$ with $C^{\infty}$ boundary $\partial \Omega^{\prime}$. Then there is a constant $C$ such that, for $u \in H^{r}\left(\Omega^{\prime}\right)$ and $u_{h} \in S_{h}\left(\Omega^{\prime}\right)$ its $A_{\Omega^{\prime}}$-projection

$$
\left\|u-u_{h}\right\|_{-r+2, \Omega^{\prime}} \leqslant C h^{2 r-2}\|u\|_{r, \Omega^{\prime}}
$$

Proof. Our task is to show that, for some constant $C$ and any $w \in C^{\infty}\left(\Omega^{\prime}\right)$, we have

$$
\left(u-u_{h}, w\right)_{\Omega^{\prime}} \leqslant C h^{2 r-2}\|u\|_{r, \Omega^{\prime}}\|w\|_{r-2, \Omega^{\prime}}
$$

Define $W$ by

$$
A(v, W)_{\Omega^{\prime}}=(v, w)_{\Omega^{\prime}} \quad \forall v \in H^{1}\left(\Omega^{\prime}\right),
$$

and recall from Lemma 2.2 that

$$
\|W\|_{r, \Omega^{\prime}} \leqslant C\|w\|_{r-2, \Omega^{\prime}}
$$

By the definition of $u_{h}$ and the continuity of $A_{\Omega^{\prime}}$, we have, for any $\chi_{h} \in S_{h}\left(\Omega^{\prime}\right)$,

$$
A\left(u-u_{h}, W\right)_{\Omega^{\prime}}=A\left(u-u_{h}, W-\chi_{h}\right)_{\Omega^{\prime}} \leqslant C\left\|u-u_{h}\right\|_{1, \Omega^{\prime}}\left\|W-\chi_{h}\right\|_{1, \Omega^{\prime}}
$$


By (8.6), (8.8), and the standard $H^{2}\left(\Omega^{\prime}\right)$ estimate

$$
\left\|u-u_{h}\right\|_{1, \Omega^{\prime}} \leqslant C \inf _{\chi_{h} \in S_{h}\left(\Omega^{\prime}\right)}\left\|u-\chi_{h}\right\|_{1, \Omega^{\prime}}
$$

easily obtained from the coercivity and continuity of $A_{\Omega^{\prime}}$, and the definition of $u_{h}$, we have

$$
\begin{aligned}
\left(u-u_{h}, w\right)_{\Omega^{\prime}} & =A\left(u-u_{h}, W\right)_{\Omega^{\prime}} \\
& \leqslant C \inf _{\chi_{h} \in S_{h}\left(\Omega^{\prime}\right)}\left\|u-\chi_{h}\right\|_{1, \Omega^{\prime}} \inf _{\chi_{h} \in S_{h}\left(\Omega^{\prime}\right)}\left\|W-\chi_{h}\right\|_{1, \Omega^{\prime}} .
\end{aligned}
$$

The desired estimate (8.5) then follows by (8.2) of Proposition 8.1 and by the regularity result (8.7).

9. High-Order Convergence for $u$ Only Locally in $W_{\infty}^{r}$. Here we shall relax the regularity assumptions on $u$ away from the point $x_{0}$ and show that $O\left(h^{2 r-2}\right)$ convergence of $\left(u-u_{h}\right)\left(x_{0}\right)$ can still hold. This will be done by localizing the arguments used in Sections 5 and 6 to a neighborhood $\Omega^{0} \subset \Omega$ of $x_{0}$. A pollution term in a so-obtained local estimate will be estimated using Theorem 8.2.

Since we consider also the case when $x_{0}$ is close to, or even on the boundary $\partial \Omega$, we shall have to introduce for $m$ a nonnegative integer and for $\Omega^{\prime} \subset \Omega$, the following special negative norm:

$$
\|v\|_{-m, \Omega^{\prime}}^{*}=\sup _{\substack{w \in C^{\infty}(\Omega) \\ \operatorname{supp}(w) \cap \overline{\Omega \backslash \Omega^{\prime}}=\varnothing}} \frac{(v, w)}{\|w\|_{m}} .
$$

Obviously, for $\Omega^{\prime} \subset \Omega^{\prime \prime}$ and $m^{\prime} \geqslant m^{\prime \prime}$, we have

$$
\|v\|_{-m^{\prime}, \Omega^{\prime}}^{*} \leqslant\|v\|_{-m^{\prime \prime}, \Omega^{\prime \prime}}^{*}
$$

and our new norm is related to those defined in Section 1 by

$$
\|v\|_{-m, \Omega^{\prime}} \leqslant\|v\|_{-m, \Omega^{\prime}}^{*} \leqslant\|v\|_{-m, \Omega^{\prime}}
$$

Besides, if $\bar{\Omega}^{\prime} \subset \Omega$ or $m=0$, then

$$
\|v\|_{-m, \Omega^{\prime}}^{*}=\|v\|_{-m, \Omega^{\prime}}
$$

We are now ready to state the announced result in precise terms.

THEOREM 9.1. Let the domain $\Omega$, the bilinear form $A$, and the family of finite-dimensional spaces $S_{h}=S_{h}\left(r, x_{0}, \alpha\right)$ be as before. Let $\Omega^{0}$ be a subdomain of $\Omega$ with $\operatorname{dist}\left(x_{0}, \Omega \backslash \Omega^{0}\right)=c_{0}>0$, and let $m \geqslant 0$ be an integer. Then, for given $\alpha$ with $(r-2) /(2 r-2)<\alpha<1$, there is a constant $C$ such that, if $u \in W_{\infty}^{r}\left(\Omega^{0}\right)$ and $u_{h} \in S_{h}\left(\Omega^{0}\right)$ satisfy

$$
A\left(u-u_{h}, \chi_{h}\right)=0 \quad \forall \chi_{h} \in S_{h}^{0}\left(\Omega^{0}\right)
$$

then

$$
\left|\left(u-u_{h}\right)\left(x_{0}\right)\right| \leqslant C h^{2 r-2}\|u\|_{W_{\infty}^{r}\left(\Omega^{0}\right)}+C\left\|u-u_{h}\right\|_{-m, \Omega^{0}}^{*}
$$

In particular, if $u \in H^{r}$ and $u_{h} \in S_{h}$ is its A-projection, then

$$
\left|\left(u-u_{h}\right)\left(x_{0}\right)\right| \leqslant C h^{2 r-2}\left(\|u\|_{W_{\infty}^{r}\left(\Omega^{0}\right)}+\|u\|_{r}\right) .
$$

If $\alpha=(r-2) /(2 r-2)$, then (9.1) and (9.2) hold with $C$ replaced by $C \ln \left(h^{-1}\right)$. 
Remark. The constant $C$ in (9.1) and (9.2) depends on $c_{0}$, but is, in fact, independent of $x_{0}$.

Below we present two lemmas which will be used in the proof of Theorem 9.1. These lemmas are proved at the end of this section. For $A_{\Omega^{0}}$ we shall use the shorter notation $A_{0}$. In the proof of Theorem 9.1 we shall estimate the two terms $\left(U-U_{h}\right)\left(x_{0}\right)$ and $\left(U_{h}-u_{h}\right)\left(x_{0}\right)$, where $U_{h} \in S_{h}\left(\Omega^{0}\right)$ is the $A_{0}$-projection of $U$ $=\left.u\right|_{\Omega^{0}}$. The term $\left(U-U_{h}\right)\left(x_{0}\right)$ will be estimated following essentially the proof of Theorem 5.1. However, for this we shall need the following analog of Lemma 6.2:

Lemma 9.2. Let $\Omega^{0}$ be as in Theorem 9.1 and have a $C^{\infty}$ boundary $\partial \Omega^{0}$, and let $j_{0}$ be the smallest integer for which $\Omega_{j_{0}} \subset \Omega^{0}$, where $\Omega_{j_{0}}=B_{j_{0}} \cap \Omega$ as before. Set $D^{\prime \prime}=$ $\Omega^{0} \backslash \bar{\Omega}_{j_{0}+2}$ and $D^{\prime}=\Omega^{0} \backslash \bar{\Omega}_{j_{0}+1}$. Then there is a constant $C$ such that, if $G \in H^{r}\left(D^{\prime \prime}\right)$ and $G_{h} \in S_{h}\left(\Omega^{0}\right)$ satisfies $A_{0}\left(\chi_{h}, G-G_{h}\right)=0 \forall \chi_{h} \in S_{h}\left(\Omega^{0}\right)$, such that $\chi_{h}=0$ on $\Omega^{0} \backslash D^{\prime \prime}$, then

$$
\left\|G-G_{h}\right\|_{1, D^{\prime}} \leqslant C h^{r-1}\|G\|_{r, D^{\prime \prime}}+C\left\|G-G_{h}\right\|_{0, D^{\prime \prime}}
$$

In order to estimate the term $\left(U_{h}-u_{h}\right)\left(x_{0}\right)$, we shall use the following result:

LEMMA 9.3. Let $\Omega^{0}$ be as in Theorem 9.1, and let $m^{\prime} \geqslant 0$ be an integer. Then there is a constant $C$ such that, if $v_{h} \in S_{h}\left(\Omega^{0}\right)$ satisfies $A\left(v_{h}, \chi_{h}\right)=0 \forall \chi_{h} \in S_{h}^{0}\left(\Omega^{0}\right)$, then

$$
\left|v_{h}\left(x_{0}\right)\right| \leqslant C\left\|v_{h}\right\|_{-m^{\prime}, \Omega^{0}}^{*} .
$$

Proof of Theorem 9.1. We may assume, without loss of generality, that $\partial \Omega^{0}$ is smooth. Set $U=\left.u\right|_{\Omega^{0}}$ and let $U_{h} \in S_{h}\left(\Omega^{0}\right)$ be its $A_{0}$-projection. Then on $\Omega^{0}$,

$$
u-u_{h}=(u-U)+\left(U-U_{h}\right)+\left(U_{h}-u_{h}\right)=0+e_{1}+e_{2} \text {. }
$$

We first claim that

$$
\left|e_{1}\left(x_{0}\right)\right|=\left|\left(U-U_{h}\right)\left(x_{0}\right)\right| \leqslant C h^{2 r-2}\|U\|_{W_{\infty}^{r}\left(\Omega^{0}\right)},
$$

where $C$ is to be replaced by $C \ln \left(h^{-1}\right)$ in the case $\alpha=(r-2) /(2 r-2)$. This is proved in the same way as Theorem 5.1 , but, instead of the subdomains $\Omega_{J+1}, D_{J}$, $D_{J-1}, \ldots$ of $\Omega$, we now consider $\Omega^{0}$ subdivided into the domains $\Omega_{J+1}, D_{J}, \ldots, D_{j_{0}+1}$, and $D^{\prime}$. The approximation properties needed for the spaces $S_{h}\left(\Omega^{0}\right)$ are given by Proposition 8.1, and the required counterpart of Lemma 6.2 for the domain $D^{\prime}$ is given by Lemma 9.2 .

We shall next show that

$$
\left|e_{2}\left(x_{0}\right)\right| \leqslant C h^{2 r-2}\|U\|_{r, \Omega^{0}}+C\left\|u-u_{h}\right\|_{-m, \Omega^{0}}^{*} .
$$

Since $U=u$ on $\Omega^{0}$, and since $\|\cdot\|_{r, \Omega^{0}} \leqslant C\|\cdot\|_{W_{\infty}^{r}\left(\Omega^{0}\right)},(9.7)$ together with (9.5) and (9.6) will show (9.1). In order to obtain (9.7), set $m^{\prime}=\max (m, r-2)$ and note that, for any $\chi_{h} \in S_{h}^{0}\left(\Omega^{0}\right)$, we have

$$
A\left(e_{2}, \chi_{h}\right)=A\left(u-u_{h}, \chi_{h}\right)-A_{0}\left(U-U_{h}, \chi_{h}\right)=0-0=0 .
$$

Hence, by Lemma 9.3,

$$
\left|e_{2}\left(x_{0}\right)\right| \leqslant C\left\|e_{2}\right\|_{-m^{\prime}, \Omega^{0}}^{*}
$$

Using first the triangle inequality and the fact that $U=u$ on $\Omega^{0}$, and then that $\|\cdot\|_{-m^{\prime}, \Omega^{0}}^{*}$ is dominated by both $\|\cdot\|_{-m, \Omega^{0}}^{*}$ and $\|\cdot\| \|_{-r+2, \Omega^{0}}$, we have

$$
\left\|e_{2}\right\|_{-m^{\prime}, \Omega^{0}}^{*} \leqslant\left\|u-u_{h}\right\|_{-m, \Omega^{0}}^{*}+\left\|U-U_{h}\right\|_{-r+2, \Omega^{0}} \text {. }
$$


From here the negative-norm estimate (8.4) settles (9.7) and, thereby, (9.1). In particular, if $u \in H^{r}$ and $u_{h} \in S_{h}$ is its $A$-projection, then (9.1) with $m=r-2$, the norm inequality $\|\cdot\|_{-m, \Omega^{0}}^{*} \leqslant\|\mid \cdot\|_{-m}$, and the negative-norm estimate (8.4) together show (9.2). This completes the proof.

Remark 9.1. Since (9.1) is entirely a local estimate, it can be used to obtain estimates similar to (9.2) also in other situations where high-order negative-norm estimates for $u-u_{h}$ are known, such as, in connection with various procedures for treating the Dirichlet problem.

Proof of Lemma 9.2. We introduce for the proof domains $D^{(j)}, j=0, \ldots, 4$, such that $D^{\prime}=D^{(0)} \subset D^{(1)} \subset \cdots \subset D^{(4)}=D^{\prime \prime}$ and with $\operatorname{dist}\left(D^{(j)}, \Omega^{0} \backslash D^{(j+1)}\right) \geqslant c>0$. Following the proof of Lemma 6.2 given in [5], we shall first show that the desired estimate (9.3) is implied by the statement: There is a constant $C$ such that, if $v \in H^{1}\left(D^{(3)}\right)$ and $v_{h} \in S_{h}\left(\Omega^{0}\right)$ satisfy $A_{0}\left(\chi_{h}, v-v_{h}\right)=0 \forall \chi_{h} \in S_{h}\left(\Omega^{0}\right)$, such that $\chi_{h}=0$ on $\Omega^{0} \backslash D^{(3)}$, then

$$
\left\|v-v_{h}\right\|_{1, D^{\prime}} \leqslant C\|v\|_{1, D^{(3)}}+C\left\|v-v_{h}\right\|_{0, D^{(3)}} .
$$

For, set $v=G-\chi_{h}$ and $v_{h}=G_{h}-\chi_{h}$, where $\chi_{h} \in S_{h}\left(\Omega^{0}\right)$ approximates $G$. Then by (9.8) and Proposition 8.1,

$$
\begin{aligned}
\left\|G-G_{h}\right\|_{1, D^{\prime}} & =\left\|G-\chi_{h}-\left(G_{h}-\chi_{h}\right)\right\|_{1, D^{\prime}} \leqslant C\left\|G-\chi_{h}\right\|_{1, D^{(3)}}+C\left\|G-G_{h}\right\|_{0, D^{(3)}} \\
& \leqslant C h^{r-1}\|G\|_{r, D^{\prime \prime}}+C\left\|G-G_{h}\right\|_{0, D^{\prime \prime}},
\end{aligned}
$$

which shows (9.3).

For the proof of (9.8) we let $\eta$ be a smooth cutoff function, vanishing in $\Omega^{0} \backslash D^{(3)}$, equal to 1 on $D^{(2)}$, and with $\|\eta\|_{W_{\infty}^{1}} \leqslant C_{\eta}$. We use the notation $(\eta v)_{h}$ for the $A_{0}$-projection of $\eta v$. By the triangle inequality, we have

$$
\left\|v-v_{h}\right\|_{1, D^{\prime}} \leqslant\left\|\eta v-(\eta v)_{h}\right\|_{1, D^{\prime}}+\left\|(\eta v)_{h}-v_{h}\right\|_{1, D^{\prime}}=I^{\prime}+I^{\prime \prime} .
$$

Using the boundedness in $H^{1}\left(\Omega^{0}\right)$ of the $A_{0}$-projection and the properties of $\eta$, we have

$$
I^{\prime} \leqslant C\|\eta v\|_{1, \Omega^{0}} \leqslant C\|v\|_{1, D^{(3)}} .
$$

In order to estimate $I^{\prime \prime}$, we need the following result: There is a constant $C$ such that, if $w_{h} \in S_{h}\left(\Omega^{0}\right)$ satisfies $A_{0}\left(\chi_{h}, w_{h}\right)=0 \forall \chi_{h} \in S_{h}\left(\Omega^{0}\right)$, such that $\chi_{h}=0$ on $\Omega^{0} \backslash D^{(2)}$, then

$$
\left\|w_{h}\right\|_{1, D^{\prime}} \leqslant C\left\|w_{h}\right\|_{0, D^{(2)}} .
$$

Using (9.9) with $w_{h}=(\eta v)_{h}-v_{h}$, we have

$$
I^{\prime \prime} \leqslant C\left\|(\eta v)_{h}-v_{h}\right\|_{0, D^{(2)}} \leqslant C\left\|v-v_{h}\right\|_{0, D^{(3)}}+C\left\|\eta v-(\eta v)_{h}\right\|_{0, \Omega^{0}},
$$

where we have also used the triangle inequality and taken larger domains in the last step. Again using the boundedness of the $A_{0}$-projection and the properties of $\eta$, we have

$$
\left\|\eta v-(\eta v)_{h}\right\|_{0, \Omega^{0}} \leqslant\left\|\eta v-(\eta v)_{h}\right\|_{1, \Omega^{0}} \leqslant C\|\eta v\|_{1, \Omega^{0}} \leqslant C\|v\|_{1, D^{(3)}},
$$

which completes the proof of (9.8).

We shall now show (9.9). Let $D_{h}^{\prime}$ and $D_{h}^{\prime \prime}$ be mesh domains on $\Omega^{0}$ such that $D^{\prime} \subset D_{h}^{\prime}, \Omega^{0} \backslash D^{(1)} \subset D_{h}^{\prime \prime}$, and $\operatorname{dist}\left(\hat{D}_{h}^{\prime}, \hat{D}_{h}^{\prime \prime}\right) \geqslant c>0$. Let $\eta$ be a new smooth cutoff 
function equal to 1 on $\hat{D}_{h}^{\prime}$, vanishing on $\hat{D}_{h}^{\prime \prime}$, and satisfying $\|\eta\|_{W_{\infty}^{\prime}} \leqslant C_{\eta}$. As in the proof of Lemma 6.2 given in [5], we have

$$
c_{A}\left\|w_{h}\right\|_{1, D^{\prime}}^{2} \leqslant c_{A}\left\|\eta w_{h}\right\|_{1, \Omega^{0}}^{2} \leqslant A_{0}\left(\eta w_{h}, \eta w_{h}\right)=A_{0}\left(\eta^{2} w_{h}, w_{h}\right)+I,
$$

where $I$ can be estimated by

$$
\frac{1}{2} c_{A}\left\|\eta w_{h}\right\|_{1, \Omega^{0}}^{2}+C\left\|w_{h}\right\|_{0, D^{(2)}}^{2} .
$$

Hence,

$$
\left\|w_{h}\right\|_{1, D^{\prime}}^{2} \leqslant C A_{0}\left(\eta^{2} w_{h}, w_{h}\right)+C\left\|w_{h}\right\|_{0, D^{(2)}}^{2} .
$$

Using the discrete $A_{0}^{*}$-harmonicity of $w_{h}$ and Proposition 4.3, we have, for some $\chi_{h} \in S_{h}\left(\Omega^{0}\right)$,

$$
A_{0}\left(\eta^{2} w_{h}, w_{h}\right)=A_{0}\left(\eta^{2} w_{h}-\chi_{h}, w_{h}\right) \leqslant C h\left\|w_{h}\right\|_{1, D^{(1)}}^{2} .
$$

Now let $D_{h}^{\prime}$ and $D_{h}^{\prime \prime}$ be new mesh domains with $D^{(1)} \subset D_{h}^{\prime}, \Omega^{0} \backslash D^{(2)} \subset D_{h}^{\prime \prime}$, and $\operatorname{dist}\left(\hat{D}_{h}^{\prime}, \hat{D}_{h}^{\prime \prime}\right) \geqslant c>0$, and let $\eta$ be a new smooth cutoff function equal to 1 on $\hat{D}_{h}^{\prime}$, vanishing on $\hat{D}_{h}^{\prime \prime}$, and satisfying $\|\eta\|_{W_{x}^{\prime}} \leqslant C_{\eta}$. Set $D_{h}=\Omega^{0} \backslash\left(\bar{D}_{h}^{\prime} \cup \bar{D}_{h}^{\prime \prime}\right)$. Repeating the above arguments then shows that, for any $\chi_{h} \in S_{h}\left(\Omega^{0}\right)$ such that $\chi_{h}=0$ on $\Omega^{0} \backslash D^{(2)}$, we have

$$
\left\|w_{h}\right\|_{1, D^{(1)}}^{2} \leqslant C A_{0}\left(\eta^{2} w_{h}-\chi_{h}, w_{h}\right)+C\left\|w_{h}\right\|_{0, D^{(2)}}^{2} .
$$

By Proposition 4.3 we have, for a suitable choice of $\chi_{h}$,

$$
A_{0}\left(\eta^{2} w_{h}-\chi_{h}, w_{h}\right) \leqslant C h\left\|w_{h}\right\|_{1, D_{h}}^{2} .
$$

Collecting the above estimates and using an inverse estimate on $D_{h}$, we have

$$
\left\|w_{h}\right\|_{1, D^{\prime}}^{2} \leqslant C h^{2}\left\|w_{h}\right\|_{1, D_{h}}^{2}+C\left\|w_{h}\right\|_{0, D^{(2)}}^{2} \leqslant C\left\|w_{h}\right\|_{0, D^{(2)}}^{2},
$$

which shows (9.9) and completes the proof of Lemma 9.2.

Proof of Lemma 9.3. Given an integer $m^{\prime} \geqslant 0$, we shall prove the existence of a constant $C$ such that, for $v_{h} \in S_{h}\left(\Omega^{0}\right)$ satisfying $A\left(v_{h}, \chi_{h}\right)=0 \forall \chi_{h} \in S_{h}^{0}\left(\Omega^{0}\right)$, we have

$$
\left|v_{h}\left(x_{0}\right)\right| \leqslant C\left\|v_{h}\right\|_{-m^{\prime}, \Omega^{0}}^{*} .
$$

Recall the definition $D_{j}^{k}=D_{j-k} \cup \cdots \cup D_{j+k}$. Set $m=\max \left(m^{\prime},[N / 2]+1\right)$, where [ $N / 2$ ] denotes the integral part of $N / 2$, and let $l$ be the smallest integer for which $D_{l}^{m+3} \subset \Omega^{0}$. With $\tilde{D}=D_{l}^{1}$ we shall show the desired estimate in two steps: namely,

$$
\left|v_{h}\left(x_{0}\right)\right| \leqslant C\left\|v_{h}\right\|_{1, \tilde{D}}
$$

and

$$
\left\|v_{h}\right\|_{1, \tilde{D}} \leqslant C\left\|v_{h}\right\|_{-m^{\prime}, \Omega^{0}}^{*} .
$$

Set $\tilde{D}=D_{l}^{m+3}$. We shall use the fact that $\tilde{\tilde{D}}$ is well separated from the point $x_{0}$, so that the mesh size on all of $\tilde{\tilde{D}}$ is of order $h$, and $d_{j}^{-1} \leqslant C$ for each $D_{j} \subset \tilde{\tilde{D}}$. For the proof of (9.10) and (9.11), 'we shall use the following proposition:

Proposition 9.4. Under the assumptions of Theorem 9.1 and with $l$ and $m$ as above, there is a constant $C$ such that, if $v_{h} \in S_{h}\left(\Omega^{0}\right)$ satisfies $A\left(v_{h}, \chi_{h}\right)=0, \chi_{h} \in S_{h}^{0}\left(D_{l}^{m+2}\right)$, 
then, for $k=1, \ldots, m+1$,

$$
\left\|v_{h}\right\|_{1, D_{l}^{k}} \leqslant C h\left\|v_{h}\right\|_{1, D_{l}^{k+1}}+C\left\|v_{h}\right\|_{-m, D_{l}^{k+1}}^{*} .
$$

Estimate (9.12) also holds for $v_{h}$ satisfying $A\left(\chi_{h}, v_{h}\right)=0 \forall \chi_{h} \in S_{h}^{0}\left(D_{l}^{m+2}\right)$.

Before proving Proposition 9.4, we use it to first prove (9.11) and then (9.10).

Repeated use of (9.12) with $k=1, \ldots, m+1$ shows that

$$
\left\|v_{h}\right\|_{1, D_{l}^{1}} \leqslant C h^{m+1}\left\|v_{h}\right\|_{1, D_{l}^{m+2}}+C\left\|v_{h}\right\|_{-m, D_{l}^{m+2}}^{*} .
$$

Let $D_{h}$ be a mesh domain such that $D_{l}^{m+2} \subset D_{h} \subset \tilde{\tilde{D}}$. By (4.5) we have

$$
\left\|v_{h}\right\|_{1, D_{l}^{m+2}} \leqslant C h^{-m-1}\left\|v_{h}\right\|_{-m, \tilde{\tilde{D}}}^{*} \leqslant C h^{-m-1}\left\|v_{h}\right\|_{-m, \tilde{\tilde{D}}}^{*},
$$

and, hence, since $m \geqslant m^{\prime}$ and $\tilde{\tilde{D}} \subset \Omega^{0}$,

$$
\left\|v_{h}\right\|_{1, \tilde{D}}=\left\|v_{h}\right\|_{1, D_{l}^{1}} \leqslant C\left\|v_{h}\right\|_{-m, \tilde{\tilde{D}}}^{*} \leqslant C\left\|v_{h}\right\|_{-m^{\prime}, \Omega^{0}}^{*}
$$

which is inequality (9.11).

In order to show (9.10), let $z_{h} \in S_{h}^{0}\left(\Omega_{l-1}\right)$ be equal to $v_{h}$ on $\Omega_{l}$ and satisfy $\left\|z_{h}\right\|_{1, \tilde{D}} \leqslant C\left\|v_{h}\right\|_{1, \tilde{D}}$. To see that such a $z_{h}$ exists, let $D_{h}^{\prime}$ and $D_{h}^{\prime \prime}$ be mesh domains such that $\Omega_{l} \subset D_{h}^{\prime}, \Omega^{0} \backslash \Omega_{l-1} \subset D_{h}^{\prime \prime}$, and with $\operatorname{dist}\left(\hat{D}_{h}^{\prime}, \hat{D}_{h}^{\prime \prime}\right) \geqslant c>0$, and let $\eta$ be a smooth cutoff function equal to 1 on $\hat{D}_{h}^{\prime}$, vanishing on $\hat{D}_{h}^{\prime \prime}$, and satisfying $\|\eta\|_{W_{\infty}^{r}} \leqslant C_{\eta}$. Take $z_{h}$ as the function $\chi_{h}$ which approximates $\eta v_{h}$, as in Proposition 4.3. The triangle inequality and $d_{l}^{-1} \leqslant C$ then give

$$
\begin{aligned}
\left\|z_{h}\right\|_{1, \tilde{D}} & \leqslant\left\|\eta v_{h}-z_{h}\right\|_{1, \tilde{D}}+\left\|\eta v_{h}\right\|_{1, \tilde{D}} \\
& \leqslant C h_{l} d_{l}^{-1}\left\|v_{h}\right\|_{1, \tilde{D}}+C h_{l} d_{l}^{-2}\left\|v_{h}\right\|_{0, \tilde{D}}+C d_{l}^{-1}\left\|v_{h}\right\|_{1, \tilde{D}} \leqslant C\left\|v_{h}\right\|_{1, \tilde{D}} .
\end{aligned}
$$

As before, let $\tau_{0}$ be an element for which $x_{0} \in \bar{\tau}_{0}$. Using the inverse estimate (4.1) and the assumption that $\operatorname{diam}\left(\tau_{0}\right)$ is of order $h^{1 /(1-\alpha)}$, we have

$$
\left|v_{h}\left(x_{0}\right)\right|=\left|z_{h}\left(x_{0}\right)\right| \leqslant C \operatorname{diam}^{-N / 2}\left(\tau_{0}\right)\left\|z_{h}\right\|_{0, \tau_{0}} \leqslant C h^{-N /(2-2 \alpha)}\left\|z_{h}\right\|_{0, \tau_{0}} .
$$

Duality gives

$$
\left\|z_{h}\right\|_{0, \tau_{0}}=\sup \left(z_{h}, g\right) h^{N / 2-2 \alpha)}
$$

where the supremum is taken over all $g \in C_{0}^{\infty}\left(\tau_{0}\right)$ normalized so that $\|g\|_{0}=$ $h^{-N / 12-2 \alpha)}$. Let $G \in H^{1}$ be defined by

$$
A(v, G)=(v, g) \quad \forall v \in H^{1},
$$

and let $G_{h} \in S_{h}$ be the $A^{*}$-projection of $G$. Let $\chi_{h} \in S_{h}^{0}\left(\Omega_{l}\right)$ equal $G_{h}$ on $\Omega_{l+1}$ and satisfy

$$
\left\|G_{h}-\chi_{h}\right\|_{1, \tilde{D}} \leqslant C\left\|G_{h}\right\|_{1, \tilde{D}} .
$$

The existence of such a $\chi_{h}$ is an easy consequence of Proposition 4.3. Since $v_{h}$ is $A$-harmonic on $\Omega^{0}$, and hence $z_{h}$ on $\Omega_{l}$, we have, by the definition of $\chi_{h}$ and $G_{h}$,

$$
\begin{aligned}
A\left(z_{h}, G\right) & =A\left(z_{h}, G-\chi_{h}\right)=A\left(z_{h}, G-G_{h}\right)+A\left(z_{h}, G_{h}-\chi_{h}\right) \\
& =A\left(z_{h}, G_{h}-\chi_{h}\right) .
\end{aligned}
$$

Hence, by the definition of $G$ and the estimates on $G_{h}-\chi_{h}$ and $z_{h}$,

$$
\begin{aligned}
\left(z_{h}, g\right) & =A\left(z_{h}, G\right)=A\left(z_{h}, G_{h}-\chi_{h}\right) \leqslant C\left\|z_{h}\right\|_{1, \tilde{D}}\left\|G_{h}-\chi_{h}\right\|_{1, \tilde{D}} \\
& \leqslant C\left\|v_{h}\right\|_{1, \tilde{D}}\left\|G_{h}\right\|_{1, \tilde{D}},
\end{aligned}
$$


where we also used that either $z_{h}$ or $G_{h}-\chi_{h}$ vanish outside $\tilde{D}$. Now note that $G_{h}$ satisfies

$$
A\left(\chi_{h}, G_{h}\right)=A\left(\chi_{h}, G\right)=\left(\chi_{h}, g\right)=0 \quad \forall \chi_{h} \in S_{h}^{0}\left(\Omega \backslash \tau_{0}\right) .
$$

Hence, Proposition 9.4 applies, and we obtain, as in (9.14),

$$
\left\|G_{h}\right\|_{1, \tilde{D}} \leqslant C\left\|G_{h}\right\|_{-m, \tilde{\tilde{D}}}^{*}
$$

We shall now use the fact that the last negative norm is dominated by the $L_{1}$-norm over $\tilde{\tilde{D}}$. Namely, since $m \geqslant[N / 2]+1$, the Sobolev inequality (cf., e.g., [1])

$$
\|w\|_{L_{\infty}} \leqslant C\|w\|_{[N / 2]+1} \leqslant C\|w\|_{m}
$$

yields

$$
\begin{aligned}
& \left\|G_{h}\right\|_{-m, \tilde{\tilde{D}}}^{*}=\sup _{\substack{w \in C^{\infty}(\Omega) \\
\operatorname{supp}(w) \cap \Omega(\tilde{\tilde{D}}=\varnothing}} \frac{\left(G_{h}, w\right)}{\|w\|_{m}} \leqslant \sup _{w \in C^{\infty}(\Omega)} \frac{\left\|G_{h}\right\|_{L_{1}(\tilde{\tilde{D}})}\|w\|_{L_{\infty}}}{\|w\|_{m}} \\
& \leqslant C\left\|G_{h}\right\|_{L_{1}(\tilde{\tilde{D}})} \leqslant C\|G\|_{L_{1}(\tilde{\tilde{D}})}+C\left\|G-G_{h}\right\|_{W_{1}^{1}(\tilde{\tilde{D}})},
\end{aligned}
$$

where we have also used the triangle inequality and changed to a sharper norm in the last step. Using Lemma 2.3 and recalling that $g$, as defined above, has bounded $L_{1}$-norm, we at once have

$$
\|G\|_{L_{1}(\tilde{\tilde{D}})} \leqslant C\|g\|_{L_{1}\left(\tau_{0}\right)} \leqslant C .
$$

For the other term we use Lemma 5.2 to obtain

$$
\begin{aligned}
\left\|G-G_{h}\right\|_{W_{1}^{1}(\tilde{\tilde{D}})} & =\left\|G-G_{h}\right\|_{W_{1}^{1}\left(D_{l}^{m+3}\right)} \leqslant h_{l+m+3}^{-r+1}\left\{\sum_{j=l-m-3}^{l+m+3} h_{j}^{r-1}\left\|G-G_{h}\right\|_{W_{1}^{1}\left(D_{j}\right)}\right\} \\
& \leqslant C h^{-r+1} \ln \left(h^{-1}\right) h^{2 r-2} \leqslant C .
\end{aligned}
$$

Collecting the above inequalities, we have shown that $\left\|G_{h}\right\|_{1, \tilde{D}} \leqslant C$, and, hence, by (9.15)-(9.17), obtained the desired estimate (9.10) and thereby proved Lemma 9.2.

Proof of Proposition 9.4. We shall prove that if $v_{h}$ is discrete $A$-harmonic in $D_{l}^{m+2}$, then, for $k=1, \ldots, m+1$,

$$
\left\|v_{h}\right\|_{1, D_{l}^{k}} \leqslant C h\left\|v_{h}\right\|_{1, D_{l}^{k+1}}+C\left\|v_{h}\right\|_{-m, D_{l}^{k+1}}^{*}
$$

Fix $k$ and introduce domains $D^{j}$ such that

$$
D_{l}^{k}=D^{-1} \subset D^{0} \subset D^{1} \subset \cdots \subset D^{m-1} \subset D^{m}=D_{l}^{k+1}
$$

and with $\operatorname{dist}\left(D^{j}, \Omega \backslash D^{j+1}\right) \geqslant c>0$ for $j=-1, \ldots, m-1$. By Lemma 7 in [5] (with $v \equiv 0)$ the $H^{1}$-norm of $v_{h}$ over $D_{j}$ is dominated by $C d_{j}^{-1}$ times the $L_{2}$-norm of $v_{h}$ over a slightly larger domain. Using the same arguments step by step, we here obtain

$$
\left\|v_{h}\right\|_{1, D_{l}^{k}} \leqslant C\left\|v_{h}\right\|_{0, D^{0}}
$$

We shall next show that, for $j=0, \ldots, m-1$, we have

$$
\left\|v_{h}\right\|_{-j, D^{j}}^{*} \leqslant C h\left\|v_{h}\right\|_{1, D^{j+1}}+C\left\|v_{h}\right\|_{-(j+1), D^{j+1}}^{*}
$$


Repeated use of $(9.20)$ for these $j$ 's yields

$$
\overline{\|} v_{h}\left\|_{0, D^{0}}^{*} \leqslant C h\right\| v_{h}\left\|_{1, D^{m}}+C\right\| v_{h} \|_{-m, D^{m}}^{*} .
$$

Since $\|\cdot\|_{0, D^{0}}=\|\cdot\|_{0, D^{0}}^{*}$, we will then have obtained the desired estimate (9.12).

In order to show (9.20), fix $j$ and consider a domain $D$ with $D^{j} \subset D \subset D^{j+1}$, $\operatorname{dist}\left(D^{j}, \Omega \backslash D\right) \geqslant c>0$, and $\operatorname{dist}\left(D, \Omega \backslash D^{j+1}\right) \geqslant c>0$. Let $\eta \in C^{\infty}\left(R^{N}\right)$ equal 1 on $D^{j}$, equal 0 on $\Omega \backslash D$, and satisfy $\|\eta\|_{W_{x}^{m}} \leqslant C_{\eta}$. Moreover, let $\eta$ satisfy the boundary condition

$$
\frac{\partial \eta}{\partial n_{c}}=\sum_{i, j=1}^{N} a_{i j} \frac{\partial \eta}{\partial x_{j}} n_{i}=0 \quad \text { on } \partial \Omega,
$$

where $n=\left(n_{i}\right)$ is the exterior normal to $\partial \Omega$. That such a function $\eta$ exists follows from the ellipticity condition (1.3). For let $\eta^{\prime}$ satisfy all requirements on $\eta$ except for the boundary condition, and let $\omega$ be a smooth function vanishing on $\partial \Omega$, with nonvanishing gradient on $\operatorname{supp}\left(\eta^{\prime}\right)$ and gradient parallel to $n$ on $\partial \Omega \cap \operatorname{supp}\left(\eta^{\prime}\right)$. Then, with $\partial_{j}=\partial / \partial x_{j}$, the function

$$
\eta=\eta^{\prime}-\frac{\sum_{i, j=1}^{N} a_{i j} \partial_{i} \omega \partial_{j} \eta^{\prime}}{\sum_{i, j=1}^{N} a_{i j} \partial_{i} \omega \partial_{j} \omega} \omega
$$

satisfies all requirements, including the boundary condition.

Now, let $w \in C^{\infty}(\Omega)$ with $\operatorname{supp}(w) \cap \overline{\Omega \backslash D^{j}}=\varnothing$, as in the definition of the negative norm $\|\cdot\|_{-j, D^{j}}^{*}$. For such $w$ define $W$ by

$$
A(v, W)=(v, w) \quad \forall v \in H^{1} .
$$

We shall integrate by parts and use the boundary condition on $\eta$. We have

$$
\begin{aligned}
\left(v_{h}, w\right)= & \left(\eta v_{h}, w\right)=A\left(\eta v_{h}, W\right) \\
= & A\left(v_{h}, \eta W\right)+\int_{\Omega} \sum_{i, j=1}^{N} a_{i j} \frac{\partial \eta}{\partial x_{i}} v_{h} \frac{\partial W}{\partial x_{j}} d x \\
& +\int_{\Omega} \sum_{i, j=1}^{N} v_{h} \frac{\partial}{\partial x_{i}}\left(a_{i j} \frac{\partial \eta}{\partial x_{j}} W\right) d x+\int_{\Omega} \sum_{i=1}^{N} a_{i} \frac{\partial \eta}{\partial x_{i}} v_{h} W d x \\
= & A\left(v_{h}, \eta W\right)+I,
\end{aligned}
$$

where

$$
I \leqslant C\left\|v_{h}\right\|_{-(j+1), D^{j+1}}^{*}\|W\|_{j+2} .
$$

Since $v_{h}$ is discrete $A$-harmonic, we have, for any $\chi_{h} \in S_{h}^{0}\left(D^{j+1}\right)$,

$$
A\left(v_{h}, \eta W\right)=A\left(v_{h}, \eta W-\chi_{h}\right) \leqslant C\left\|v_{h}\right\|_{1, D^{j+1}}\left\|\eta W-\chi_{h}\right\|_{1, D^{j+1}} .
$$

By the approximation properties obtained in Section 4 , there is such a $\chi_{h}$ with

$$
\left\|\eta W-\chi_{h}\right\|_{1, D^{j+1}} \leqslant C h\|W\|_{2} \leqslant C h\|W\|_{j+2} \text {. }
$$

Since Lemma 2.2 shows that $\|W\|_{j+2} \leqslant C\|w\|_{j}$, we have thus obtained

$$
\left(v_{h}, w\right) \leqslant C\left\{h\left\|v_{h}\right\|_{1, D^{j+1}}+\left\|v_{h}\right\|_{-(j+1), D^{j+1}}^{*}\right\}\|w\|_{j},
$$


with $C$ independent of $h, v_{h}$, and $w$ as above. Since this is the same as (9.20), and since the same estimate could be obtained for $v_{h}$ being discrete $A^{*}$-harmonic in $D_{l}^{m+2}$, we have thereby proved Proposition 9.4.

10. High-Order Convergence for Derivatives for $u$ Only Locally in $W_{\infty}^{r}$. In this section we shall prove a counterpart of the localized estimate for $\left(u-u_{h}\right)\left(x_{0}\right)$ of Section 9 for the gradient and show that $O\left(h^{2 r-2}\right)$ convergence of $\nabla\left(u-u_{h}\right)\left(x_{0}\right)$ can also hold when $u$ is only locally in $W_{\infty}^{r}$.

THEOREM 10.1. Let the domain $\Omega$, the bilinear form $A$, and the family of finitedimensional spaces $S_{h}=S_{h}\left(r, x_{0}, \alpha\right)$ be as before. Let $\Omega^{0} \subset \Omega$ and assume that $\operatorname{dist}\left(x_{0}, \Omega \backslash \Omega^{0}\right)=c_{0}>0$. Moreover, let $m \geqslant 0$ be an integer. Then, given $\alpha$ with $1 / 2<\alpha<1$, there is a constant $C$ such that, if $u \in W_{\infty}^{r}\left(\Omega^{0}\right)$ and if $u_{h} \in S_{h}\left(\Omega^{0}\right)$ satisfies $A\left(u-u_{h}, \chi_{h}\right) \forall \chi_{h} \in S_{h}^{0}\left(\Omega^{0}\right)$, then

$$
\left|\nabla\left(u-u_{h}\right)\left(x_{0}\right)\right| \leqslant C h^{2 r-2}\|u\|_{W_{\infty}^{r}\left(\Omega^{0}\right)}+C\left\|u-u_{h}\right\|_{-m, \Omega^{0}}^{*}
$$

In particular, if $u \in H^{r}$ and $u_{h} \in S_{h}$ is the A-projection of $u$, then

$$
\left|\nabla\left(u-u_{h}\right)\left(x_{0}\right)\right| \leqslant C h^{2 r-2}\left\{\|u\|_{W_{\infty}^{r}\left(\Omega^{0}\right)}+\|u\|_{r}\right\} .
$$

If $\alpha=1 / 2$ then (10.1) and (10.2) hold with $C$ replaced by $C \ln \left(h^{-1}\right)$.

Remark. The constant $C$ in (10.1) and (10.2) depends on $c_{0}$, but is independent of $x_{0}$.

For the proof of the theorem we shall need the following lemma.

LEMMA 10.2. Under the assumptions of Theorem 10.1 and given a nonnegative integer $m^{\prime}$, there is a constant $C$ such that, if $v_{h} \in S_{h}\left(\Omega^{0}\right)$ satisfies

$$
A\left(v_{h}, \chi_{h}\right)=0 \quad \forall \chi_{h} \in S_{h}^{0}\left(\Omega^{0}\right)
$$

then, for any $\partial_{j}=\partial / \partial x_{j}$,

$$
\left|\partial_{j} v_{h}\left(x_{0}\right)\right| \leqslant C\left\|v_{h}\right\|_{-m^{\prime}, \Omega^{0}}^{*}
$$

We give the necessary details of the proof of Lemma 10.2 after having shown (10.1) and (10.2).

Proof of Theorem 10.1. We may assume, without loss of generality, that $\partial \Omega^{0}$ is smooth. We shall prove (10.1) and (10.2) with $\nabla$ replaced by any $\partial_{j}$. Set $U=\left.u\right|_{\Omega^{0}}$ and let $U_{h} \in S_{h}\left(\Omega^{0}\right)$ be the $A_{0}$-projection of $U$. Then on $\Omega^{0}$,

$$
\partial_{j}\left(u-u_{h}\right)=\partial_{j}(u-U)+\partial_{j}\left(U-U_{h}\right)+\partial_{j}\left(U_{h}-u_{h}\right)=0+\partial_{j} e_{1}+\partial_{j} e_{2} .
$$

We first claim that

$$
\left|\partial_{j} e_{1}\left(x_{0}\right)\right| \leqslant C h^{2 r-2}\|U\|_{W_{\infty}^{r}\left(\Omega^{0}\right)},
$$

with $C$ replaced by $C \ln \left(h^{-1}\right)$ in the case $\alpha=1 / 2$. This is proved in the same way as Theorem 7.1, but with the modifications described after (9.6). We shall next show that

$$
\left|\partial_{j} e_{2}\left(x_{0}\right)\right| \leqslant C h^{2 r-2}\|U\|_{r, \Omega^{0}}+C\left\|u-u_{h}\right\|_{-m, \Omega^{0}}^{*}
$$


Since $U=u$ on $\Omega^{0}$, and since $\|\cdot\|_{r, \Omega^{0}} \leqslant C\|\cdot\|_{W_{\infty}^{r}\left(\Omega^{0}\right)}$, (10.7) together with (10.5) and (10.6) will show (10.1). By taking $m=r-2$ in (10.1), using $\|\cdot\|_{-m, \Omega^{0}}^{*} \leqslant\|\| \cdot \|\left.\right|_{-m, \Omega}$ and the negative-norm estimate (8.4), we obtain (10.2).

In order to show (10.7) we first note that $e_{2}$ satisfies (10.3). Therefore, with $m^{\prime}=\max (m, r-2)$, we have

$$
\left|\partial_{j} e_{2}\left(x_{0}\right)\right| \leqslant C\left\|e_{2}\right\|_{-m^{\prime}, \Omega^{0}}^{*} .
$$

Using first the triangle inequality and the fact that $U=u$ on $\Omega^{0}$, and then using the fact that $\|\cdot\|_{-m^{\prime}, \Omega^{0}}^{*}$ is dominated by both \|\|$_{-r+2, \Omega^{0}}$ and $\|\cdot\|_{-m, \Omega^{0}}^{*}$, we have

$$
\left\|e_{2}\right\|_{-m^{\prime}, \Omega^{0}}^{*} \leqslant\left\|U-U_{h}\right\|_{-r+2, \Omega^{0}}+\left\|u-u_{h}\right\|_{-m, \Omega^{0}}^{*} .
$$

From here another application of the negative-norm estimate (8.4) shows (10.7) and completes the proof of Theorem 10.1

Remark 10.1. The same comment as in Remark 9.1 applies to estimates (10.1) and (10.2).

Proof of Lemma 10.2. In view of the previously obtained estimate (9.11) for a function $v_{h}$ satisfying (10.3), it is sufficient to show that

$$
\left|\partial_{j} v_{h}\left(x_{0}\right)\right| \leqslant C\left\|v_{h}\right\|_{1, \tilde{D}},
$$

where $\tilde{D}=D_{l}^{1}$ as in the proof of Lemma 9.3. Thus, let $z_{h} \in S_{h}^{0}\left(\Omega_{l-1}\right)$ equal $v_{h}$ on $\Omega_{l}$ and satisfy

$$
\left\|z_{h}\right\|_{1, \tilde{D}} \leqslant C\left\|v_{h}\right\|_{1, \tilde{D}}
$$

By Lemma 7.2 we have

$$
\left|\partial_{j} v_{h}\left(x_{0}\right)\right|=\left|\partial_{j} z_{h}\left(x_{0}\right)\right| \leqslant C \sup \left(\partial_{j} z_{h}, g\right)=C \sup \left(z_{h}, g^{\prime}\right),
$$

where the supremum is taken over all $g \in C_{0}^{\infty}\left(\tau_{0}\right)\left(\bar{\tau}_{0} \ni x_{0}\right)$ with

$$
\operatorname{diam}^{N / 2}\left(\tau_{0}\right)\|g\|_{0}+\operatorname{diam}^{(N+2) / 2}\left(\tau_{0}\right)\|g\|_{1} \leqslant 1,
$$

and where we have also integrated by parts and put $g^{\prime}=-\partial_{j} g$ in the last step. Define $G^{\prime}$ by

$$
A\left(v, G^{\prime}\right)=\left(v, g^{\prime}\right) \quad \forall v \in H^{1},
$$

and let $G_{h}^{\prime} \in S_{h}$ be its $A^{*}$-projection. Let $\chi_{h} \in S_{h}^{0}\left(\Omega_{l}\right)$ equal $G_{h}^{\prime}$ on $\Omega_{l+1}$ and satisfy

$$
\left\|G_{h}^{\prime}-\chi_{h}\right\|_{1, \tilde{D}} \leqslant C\left\|G_{h}^{\prime}\right\|_{1, \tilde{D}} .
$$

Since either $z_{h}$ or $G_{h}^{\prime}-\chi_{h}$ vanish outside $\tilde{D}$, we have

$$
\left(z_{h}, g^{\prime}\right)=A\left(z_{h}, G^{\prime}\right)=A\left(z_{h}, G_{h}^{\prime}\right)=A\left(z_{h}, G_{h}^{\prime}-\chi_{h}\right) \leqslant C\left\|z_{h}\right\|_{1, \tilde{D}}\left\|G_{h}^{\prime}\right\|_{1, \tilde{D}}
$$

It now remains to show that the last factor can be bounded by a constant. By (9.18) and (9.19) we have

$$
\left\|G_{h}^{\prime}\right\|_{1, \tilde{D}} \leqslant C\left\|G^{\prime}\right\|_{L_{1}(\tilde{\tilde{D}})}+\left\|G^{\prime}-G_{h}^{\prime}\right\|_{W_{1}^{1}(\tilde{\tilde{D}})} .
$$

Using the representation

$$
G^{\prime}(x)=\int_{\Omega} \mathscr{G}(x, y) g^{\prime}(y) d y=\int_{\Omega} \partial_{j} \mathscr{G}(x, y) g(y) d y
$$


and estimates (2.6) and (7.5) for $\mathscr{G}$ and $g$, respectively, we easily obtain

$$
\left\|G^{\prime}\right\|_{L_{1}(\tilde{\tilde{D}})} \leqslant C \text {. }
$$

Finally, by Lemma 7.3, we have

$$
\begin{aligned}
\left\|G^{\prime}-G_{h}^{\prime}\right\|_{W_{1}^{1}(\tilde{\tilde{D}})} & =\left\|G^{\prime}-G_{h}^{\prime}\right\|_{W_{1}^{1}\left(D_{l}^{m+3}\right)} \\
& \leqslant h_{l+m+3}^{-r+1}\left\{\sum_{j=l-m-3}^{1+m+3} h_{j}^{r-1}\left\|G^{\prime}-G_{h}^{\prime}\right\|_{W_{1}^{1}\left(D_{j}\right)}\right\} \\
& \leqslant C h^{-r+1} \ln \left(h^{-1}\right) h^{2 r-2} \leqslant C,
\end{aligned}
$$

which completes the proof of Lemma 10.2.

Remark 10.3. Improved convergence holds also for higher-order derivatives of $u-u_{h}$ at $x_{0}$, provided the mesh is correspondingly further refined.

Remark 10.4. If $u$ is in $W_{\infty}^{m}, 0<m<r$, the estimate

$$
\left|\left(u-u_{h}\right)\left(x_{0}\right)\right| \leqslant C h^{m+r-2}\|u\|_{W_{x}^{m}}
$$

can be obtained for suitable mesh-refinements.

Remark 10.5. In view of (5.2) and (9.2) and the corresponding more precise estimates (7.1) and (10.2), it is natural to ask if one can further relax the requirement on $u$ and retain the $O\left(h^{2 r-2}\right)$ convergence at $x_{0}$. It is clear from approximation theory that we cannot essentially weaken the norm on $u$ near $x_{0}$ if these estimates are supposed to hold for any $\alpha>(r-2) /(2 r-2)$ and $\alpha>1 / 2$, respectively. On the other hand, it seems reasonable to believe that by a more sophisticated analysis, one could replace the norm on $u$ by one which changes continuously from a $W_{\infty}^{r}$-norm near $x_{0}$ to an $H^{r}$-norm away from $x_{0}$ in some appropriate way. However, we have no proof of this. Neither have we investigated if $O\left(h^{2 r-2}\right)$ convergence can be obtained at $x_{0}$ for some degree of refinement if $u$ is globally in $H^{r}$ and locally in $W_{\infty}^{m}$ with $m<r$.

Department of Mathematics

Chalmers University of Technology

and the University of Göteborg

S-412 96 Göteborg, Sweden

1. R. A. Adams, Sobolev Spaces. Academic Press, New York, 1975.

2. J. H. Bramble \& S. R. Hilbert, "Estimation of linear functionals on Sobolev spaces with application to Fourier transforms and spline interpolation," SIAM J. Numer. Anal., 7, 1970, pp. 112-124.

3. J. H. BRAmble \& J. E. Osborn, "Rate of convergence estimates for nonselfadjoint eigenvalue approximations," Math. Comp., v. 27, 1973, pp. 525-549.

4. J. H. Bramble \& A. H. SChatz, "Higher order local accuracy by averaging in the finite element method," Math. Comp., v. 31, 1977, pp. 94-111.

5. K. ERIKSSON, "Improved accuracy by adapted mesh-refinements in the finite element method," Math. Comp., v. 44, 1985, pp. 321-343.

6. K. ERIKSSON, "Finite element methods of optimal order for problems with singular data," Math. Comp., v. 44, 1985 , pp. $345-360$.

7. P. G. Ciarlet, The Finite Element Method for Elliptic Problems, North-Holland, Amsterdam, 1978.

8. S. R. Hilbert, "A mollifier useful for approximations in Sobolev spaces and some applications to approximating solutions of differential equations," Math. Comp., v. 27, 1973, pp. 81-89.

9. JU. P. KRASOVSKII, "Isolation of singularities of the Green's function," Math. USSR-Izv', v. 1. 1967, pp. 935-966.

10. A. Louis \& F. NAtTerer, Acceleration of Convergence for Finite Element Solutions of the Poisson Equation on Irregular Meshes, Preprint, 1977. 
11. J. A. Nitsche, $L_{\infty}$-Error Analysis for Finite Elements, Proc. 3rd Conf. on Mathematics of Finite Elements and Applications, Brunel University, 1978.

12. J. A. Nitsche \& A. H. Schatz, “Interior estimates for Ritz-Galerkin methods," Math. Comp., v. 28,1974 , pp. 937-958.

13. A. H. Schatz \& L. B. WahlBin, "Interior maximum norm estimates for finite element methods," Math. Comp., v. 31, 1977, pp. 414-442.

14. A. H. Schatz \& L. B. Wahlbin, "Maximum norm estimates in the finite element method on plane polygonal domains. Part 1,” Math. Comp., v. 32, 1978, pp. 73-109.

15. A. H. Schatz \& L. B. Wahlbin, "Maximum norm estimates in the finite element method on plane polygonal domains. Part 2: Refinements," Math. Comp., v. 33, 1979, pp. 465-492.

16. A. H. Schatz \& L. B. Wahlbin, "On the quasi-optimality in $L_{\infty}$ of the $\stackrel{H}{ }^{1}$-projection into finite element spaces,” Math. Comp., v. 38, 1982, pp. 1-22.

17. M. SChechter, “On $L^{p}$ estimates and regularity. I." Amer. J. Math., v. 85, 1963, pp. 1-13.

18. R. SCHREIBER, "Finite element methods of high-order accuracy for singular two-point boundary value problems with nonsmooth solutions," SIAM J. Numer. Anal., v. 17, 1980, pp. 547-566.

19. R. Scotr, "Optimal $L_{\infty}$ estimates for the finite element method on irregular meshes," Math. Comp., v. 30, 1976, pp. 681-697.

20. V. ThOмEe, "High order local approximations to derivatives in the finite element method," Math. Comp., v. 31, 1977, pp. 652-660. 